\title{
Nguetseng's Two-scale Convergence Method For Filtration and Seismic Acoustic Problems in Elastic Porous Media
}

\author{
Anvarbek Meirmanov*
}

\begin{abstract}
A linear system of differential equations describing a joint motion of elastic porous body and fluid occupying porous space is considered. Although the problem is linear, it is very hard to tackle due to the fact that its main differential equations involve non-smooth oscillatory coefficients, both big and small, under the differentiation operators. The rigorous justification, under various conditions imposed on physical parameters, is fulfilled for homogenization procedures as the dimensionless size of the pores tends to zero, while the porous body is geometrically periodic. As the results, we derive Biot's equations of poroelasticity, equations of viscoelasticity, or decoupled system consisting of non-isotropic Lamé's equations and Darcy's system of filtration, depending on ratios between physical parameters. The proofs are based on Nguetseng's two-scale convergence method of homogenization in periodic structures.
\end{abstract}

Key words: Biot's equations, Stokes equations, Lamé's equations, two-scale convergence, homogenization of periodic structures, poroelasticity, viscoelasticity.

\section{Introduction}

In this article a problem of modelling of small perturbations in elastic deformable medium, perforated by a system of channels (pores) filled with liquid or gas, is considered. Such media are called elastic porous media and they are a rather good approximation to real consolidated grounds. In presentday literature, the field of study in mechanics corresponding to these media is called poromechanics [1. The solid component of such a medium has a name of skeleton, and the domain, which is filled with a fluid, is named a porous space. The exact mathematical model of elastic porous medium consists of the classical equations of momentum and mass balance, which are stated in Euler variables, of the equations determining stress fields in both solid and liquid phases, and of an endowing relation determining behavior of the interface between liquid and solid components. The latter relation

*This work is partially supported by the grant of Higher Education Commission of Pakistan under National Research Program for Universities. The title of the grant is: Homogenization of Underground Flows 
expresses the fact that the interface is a material surface, which amounts to the condition that it consists of the same material particles all the time. Denoting by $\rho$ the density of medium, by $\boldsymbol{v}$ the velocity, by $\mathbb{P}^{f}$ the stress tensor in the liquid component, by $\mathbb{P}^{s}$ the stress tensor in the rigid skeleton, and by $\tilde{\chi}$ the characteristic (indicator) function of porous space, we write the fundamental differential equations of the nonlinear model in the form

$$
\begin{gathered}
\rho \frac{d \boldsymbol{v}}{d t}=\operatorname{div}_{x}\left\{\tilde{\chi} \mathbb{P}^{f}+(1-\tilde{\chi}) \mathbb{P}^{s}\right\}+\rho \boldsymbol{F}, \\
\frac{d \rho}{d t}+\rho \operatorname{div}_{x} \boldsymbol{v}=0, \quad \frac{d \tilde{\chi}}{d t}=0
\end{gathered}
$$

where $d / d t$ stands for the material derivative with respect to the time variable.

Clearly the above stated original model is a model with an unknown (free) boundary. The more precise formulation of the nonlinear problem is not in focus of our present work. Instead, we aim to study the problem, linearized at the rest state. In continuum mechanics the methods of linearization are developed rather deeply. The so obtained linear model is a commonly accepted and basic one for description of filtration and seismic acoustics in elastic porous media (see, for example, [2, 3, 4]). Further we refer to this model as to model A. In this model the characteristic function of the porous space $\tilde{\chi}$ is a known function for $t>0$. It is assumed that this function coincides with the characteristic function of the porous space $\bar{\chi}$, given at the initial moment. Being written in terms of dimensionless variables, the differential equations of the model involve frequently oscillating non-smooth coefficients, which have structures of linear combinations of the function $\bar{\chi}$. These coefficients undergo differentiation with respect to $\boldsymbol{x}$ and besides may be very big or very small quantities as compared to the main small parameter $\varepsilon$. In the model under consideration we define $\varepsilon$ as the characteristic size of pores $l$ divided by the characteristic size $L$ of the entire porous body:

$$
\varepsilon=\frac{l}{L} .
$$

Denoting by $\boldsymbol{w}$ the dimensionless displacement vector of the continuum medium, in terms of dimensionless variables we write the differential equations of model A as follows:

$$
\begin{gathered}
\alpha_{\tau} \bar{\rho} \frac{\partial^{2} \boldsymbol{w}}{\partial t^{2}}=\operatorname{div}_{x} \mathbb{P}+\bar{\rho} \boldsymbol{F} \\
\mathbb{P}=\bar{\chi} \alpha_{\mu} \mathbb{D}\left(x, \frac{\partial \boldsymbol{w}}{\partial t}\right)+(1-\bar{\chi}) \alpha_{\lambda} \mathbb{D}(x, \boldsymbol{w})-(q+\pi) \mathbb{I} \\
p=-\alpha_{p} \bar{\chi} \operatorname{div}_{x} \boldsymbol{w}, \quad \pi=-\alpha_{\eta}(1-\bar{\chi}) \operatorname{div}_{x} \boldsymbol{w}, \quad q=p+\frac{\alpha_{\nu}}{\alpha_{p}} \frac{\partial p}{\partial t} .
\end{gathered}
$$


Here and further we use the notation

$$
\bar{\rho}=\bar{\chi} \rho_{f}+(1-\bar{\chi}) \rho_{s}, \quad \mathbb{D}(x, \boldsymbol{u})=(1 / 2)\left(\nabla_{x} \boldsymbol{u}+\left(\nabla_{x} \boldsymbol{u}\right)^{T}\right) .
$$

From the purely mathematical point of view, the corresponding initialboundary value problem for model $\mathbf{A}$ is well-posed in the sense that it has a unique solution belonging to a suitable functional space on any finite temporal interval. However, in view of possible applications, for example, for developing numerical codes, this model is ineffective due to its sophistication even if a modern supercomputer is available. Therefore a question of finding an effective approximate models is vital. Since the model involves the small parameter $\varepsilon$, the most natural approach to this question is to derive models that would describe limiting regimes arising as $\varepsilon$ tends to zero. Such an approximation significantly simplifies the original problem and at the same time preserves all of its main features. But even this approach is too hard to work out, and some additional simplifying assumptions are necessary. In terms of geometrical properties of the medium, the most appropriate is to simplify the problem postulating that the porous structure is periodic. Further by model $\boldsymbol{B}^{\varepsilon}$ we will call model $\mathbf{A}$ supplemented by this periodicity condition. Thus, our main goal now is a derivation of all possible homogenized equations in the model $\boldsymbol{B}^{\varepsilon}$.

The first research with the aim of finding limiting regimes in the case when the skeleton was assumed to be an absolutely rigid body was carried out by E. Sanchez-Palencia and L. Tartar. E. Sanchez-Palencia [3, Sec. 7.2] formally obtained Darcy's law of filtration using the method of twoscale asymptotic expansions, and L. Tartar [3, Appendix] mathematically rigorously justified the homogenization procedure. Using the same method of two-scale expansions J. Keller and R. Burridge [2] derived formally the system of Biot's equations [5] from model $\boldsymbol{B}^{\varepsilon}$ in the case when the parameter $\alpha_{\mu}$ was of order $\varepsilon^{2}$, and the rest of the coefficients were fixed independent of $\varepsilon$. It is well-known that the various modifications of Biot's model are bases of seismic acoustics problems up-to-date. This fact emphasizes importance of comprehensive study of model $\mathbf{A}$ and model $\boldsymbol{B}^{\varepsilon}$ one more time. J. Keller and R. Burridge also considered model $\boldsymbol{B}^{\varepsilon}$ under assumption that all the physical parameters were fixed independent of $\varepsilon$, and formally derived as the result a system of equations of viscoelasticity.

Under the same assumptions as in the article [2], the rigorous justification of Biot's model was given by G. Nguetseng [9] and later by A. Mikelić, R. P. Gilbert, Th. Clopeaut, and J. L. Ferrin in [4, 6, 7]. Also A. Mikelić et al derived a system of equations of viscoelasticity, when all the physical parameters were fixed independent of $\varepsilon$. In these works, Nguetseng's twoscale convergence method [8, 10] was the main method of investigation of the model $\boldsymbol{B}^{\varepsilon}$. 
In the present work by means of the same method we investigate all possible limiting regimes in the model $\boldsymbol{B}^{\varepsilon}$. This method in rather simple form discovers the structure of the weak limit of a sequence $\left\{z^{\varepsilon}\right\}$ as $\varepsilon \searrow 0$, where $z^{\varepsilon}=u^{\varepsilon} v^{\varepsilon}$ and sequences $\left\{u^{\varepsilon}\right\}$ and $\left\{v^{\varepsilon}\right\}$ converge as $\varepsilon \searrow 0$ merely weakly, but at the same time function $u^{\varepsilon}$ has the special structure $u^{\varepsilon}(\boldsymbol{x})=u(\boldsymbol{x} / \varepsilon)$ with $u(\boldsymbol{y})$ being periodic in $\boldsymbol{y}$.

Moreover, Nguetseng's method allows to establish asymptotic expansions of a solution of model $B^{\varepsilon}$ in the form

$$
\boldsymbol{w}^{\varepsilon}(\boldsymbol{x}, t)=\varepsilon^{\beta}\left(\boldsymbol{w}_{0}(\boldsymbol{x}, t)+\varepsilon \boldsymbol{w}_{1}(\boldsymbol{x}, t, \boldsymbol{x} / \varepsilon)+o(\varepsilon)\right),
$$

where $\boldsymbol{w}_{0}(\boldsymbol{x}, t)$ is a solution of the homogenized (limiting) problem, $\boldsymbol{w}_{1}(\boldsymbol{x}, t, \boldsymbol{y})$ is a solution of some initial-boundary value problem posed on the generic periodic cell of the porous space, and exponent $\beta$ is defined by dimensionless parameters of the model. Distinct asymptotic behavior of these parameters and distinct geometry of the porous space lead to different limiting regimes, namely, to various forms of Darcy's law for velocity of liquid component and of non-isotropic Lamé's equations for displacement of rigid component in cases of big parameter $\alpha_{\lambda}$, also, to various forms of Biot's system in cases of small parameter $\alpha_{\mu}$, and to different forms of equations of viscoelasticity in cases when parameters $\alpha_{\mu}$ and $\alpha_{\lambda}$ are $O(1)$ as $\varepsilon \searrow 0$. For example, in the case when

$$
\alpha_{\lambda}=\varepsilon^{-\frac{3}{2}}, \quad \alpha_{\mu}=\varepsilon^{\frac{1}{2}},
$$

the velocity $\boldsymbol{v}^{\varepsilon}=\chi^{\varepsilon} \partial \boldsymbol{w}^{\varepsilon} / \partial t$ of liquid component and the displacement $\boldsymbol{u}^{\varepsilon}=$ $\left(1-\chi^{\varepsilon}\right) \boldsymbol{w}^{\varepsilon}$ of rigid skeleton possess the following asymptotic:

$$
\begin{gathered}
\boldsymbol{v}^{\varepsilon}(\boldsymbol{x}, t)=\varepsilon^{\frac{3}{2}} \chi^{\varepsilon}(\boldsymbol{V}(\boldsymbol{x}, t, \boldsymbol{x} / \varepsilon)+o(\varepsilon)), \\
\boldsymbol{u}^{\varepsilon}(\boldsymbol{x}, t)=\varepsilon^{\frac{3}{2}}\left(1-\chi^{\varepsilon}\right)(\boldsymbol{u}(\boldsymbol{x}, t)+\varepsilon \boldsymbol{U}(\boldsymbol{x}, t, \boldsymbol{x} / \varepsilon)+o(\varepsilon)) .
\end{gathered}
$$

At the same time all equations are determined in a unique way by the given physical parameters of the original model and by geometry of the porous space. For example, in the case of isolated pores (disconnected porous space) the unique limiting regime for any combinations of parameters is a regime described by the non-isotropic system of Lamé's equations.

On our opinion, the proposed approach, when the limiting transition in all coefficients is fulfilled simultaneously, is the most natural one. We emphasize that it is not assumed starting from the original model that the fluid component is inviscid, the porous skeleton is absolutely rigid, or either of the components is incompressible. These kinds of properties arise in limiting models depending on limiting relations, which involve all parameters of the problem.

The articles [4] and [6], as well as the present one, show in favor of such a uniform approach, because they exhibit the situations, when various rates 
of approach of the small parameter $\alpha_{\mu}$ to zero yield distinct homogenized equations. Moreover, these equations differ from the homogenized equations derived as the limit of model $\boldsymbol{B}^{\varepsilon}$ under assumption $\alpha_{\mu} \equiv 0$, imposed even before homogenization [7].

Suppose that all dimensionless parameters of the $\boldsymbol{B}^{\varepsilon}$ depend on the small parameter $\varepsilon$ of the model and there exist limits (finite or infinite)

$$
\lim _{\varepsilon \searrow 0} \alpha_{\mu}(\varepsilon)=\mu_{0}, \quad \lim _{\varepsilon \searrow 0} \alpha_{\lambda}(\varepsilon)=\lambda_{0}, \quad \lim _{\varepsilon \searrow 0} \alpha_{\tau}(\varepsilon)=\tau_{0}
$$

We restrict our consideration by the cases when $\tau_{0}<\infty$ and either of the following situations has place.

$$
\begin{array}{cl}
\mu_{0}=0, & 0<\lambda_{0}<\infty, \\
0 \leq \mu_{0}<\infty, & \lambda_{0}=\infty, \\
0<\mu_{0}, \lambda_{0}<\infty . &
\end{array}
$$

If $\tau_{0}=\infty$ then, re-normalizing the displacement vector by setting

$$
\boldsymbol{w} \rightarrow \alpha_{\tau} \boldsymbol{w}
$$

we reduce the problem to one of the cases $(I)-(I I I)$.

In the present paper we show that in the case $(I)$ the homogenized equations have various forms of Biot's system of equations of poroelasticity for a two-velocity continuum media or non-isotropic Lam'e's system of equations of one-velocity continuum media (for example, for the case of disconnected porous space)(theorem 2.2 ). In the case $(I I)$ the homogenized equations are different modifications of Darcy's system of equations of filtration for the velocity of the liquid component (and as a first approximation the solid component behaves yourself as an absolutely rigid body) and as a second approximation - non-isotropic Lam'e's system of equations for the re-normalized displacements of the solid component or Biot's system of equations of poroelasticity for the re-normalized displacements of the liquid and solid components(theorem 2.3). Finally, in the case (III) they are the nonlocal viscoelasticity equations or non-isotropic non-local Lam'e's system of equations of one-velocity continuum media (theorem 2.4).

\section{$\S 1$. Models $\mathbf{A}$ and $\boldsymbol{B}^{\varepsilon}$}

1.1. Differential equations, boundary and initial conditions. Let a domain $\Omega^{*}$ of the physical space $\mathbb{R}^{3}$ be the union of a domain $\Omega_{s}^{*}$ occupied with the rigid porous ground and a domain $\Omega_{f}^{*}$ corresponding to hollows (pores) in the ground. Domain $\Omega_{f}^{*}$ is called a porous space and is assumed to be filled with liquid or gas. Denote by $\boldsymbol{w}^{*}\left(\boldsymbol{x}^{*}, t^{*}\right)$ the displacement vector of 
the continuum medium (of ground or liquid or gas)) at the point $\boldsymbol{x}^{*} \in \Omega^{*}$ in Euler coordinate system at the moment of time $t^{*} \geq 0$. Under an assumption that the displacement vector $\boldsymbol{w}^{*}$ is small in $\Omega^{*}$, which amounts to a case when deformations are small, dynamics of rigid phase is described by linear Lamé's equations and dynamics of fluid or gas is described by Stokes equations. At the same time we may set that the velocity vector $\boldsymbol{v}_{f}^{*}\left(\boldsymbol{x}^{*}, t^{*}\right)$ in fluid or gas is a partial derivative of the displacement vector with respect to time variable, i.e., that $\boldsymbol{v}_{f}^{*}\left(\boldsymbol{x}^{*}, t^{*}\right)=\partial \boldsymbol{w}_{f}^{*}\left(\boldsymbol{x}^{*}, t^{*}\right) / \partial t^{*}$. This assumption makes perfect sense in description of continuous media in domains, where the characteristic size of pores $l$ is very small as compared to the diameter $L$ of the domain $\Omega^{*}$, i.e., $l=\varepsilon L$ and $\varepsilon<<1$ (see, for example, [2, 3, 4] and recall the observation of the linearization procedure for the exact nonlinear model in Introduction).

In terms of the dimensionless variables, not denoted by the asterisk * below,

$$
\left.\begin{array}{l}
\boldsymbol{x}^{*}=\boldsymbol{x} L, \quad t^{*}=t \tau, \quad \boldsymbol{w}_{f}^{*}=\boldsymbol{w}_{f} L, \quad \boldsymbol{w}_{s}^{*}=\boldsymbol{w}_{s} L, \\
\boldsymbol{F}^{*}=\boldsymbol{F} g, \quad p_{f}^{*}=p p_{0}, \quad \rho_{f}^{*}=\rho_{f} \rho_{0}, \quad \rho_{s}^{*}=\rho_{s} \rho_{0},
\end{array}\right\}
$$

the displacement $\boldsymbol{w}_{f}(\boldsymbol{x}, t)$ and the pressure $p(\boldsymbol{x}, t)$ of fluid and the displacement $\boldsymbol{w}_{s}(\boldsymbol{x}, t)$ of rigid skeleton satisfy the system of Stokes equations

$$
\begin{array}{rr}
\alpha_{\tau} \rho_{f} \frac{\partial^{2} \boldsymbol{w}_{f}}{\partial t^{2}}=\operatorname{div}_{x} \mathbb{P}^{f}+\rho_{f} \boldsymbol{F}, & \boldsymbol{x} \in \Omega_{f}, t>0,(1.2) \\
\mathbb{P}^{f}=-p \mathbb{I}+\alpha_{\nu}\left(\operatorname{div}_{x} \frac{\partial \boldsymbol{w}_{f}}{\partial t}\right) \cdot \mathbb{I}+\alpha_{\mu} \mathbb{D}\left(x, \frac{\partial \boldsymbol{w}_{f}}{\partial t}\right), & \boldsymbol{x} \in \Omega_{f}, t>0,(1.3) \\
p=-\alpha_{p} \operatorname{div}_{x} \boldsymbol{w}_{f}, & \boldsymbol{x} \in \Omega_{f}, t>0(1.4)
\end{array}
$$

and the system of Lamé's equations

$$
\begin{aligned}
\alpha_{\tau} \rho_{s} \frac{\partial^{2} \boldsymbol{w}_{s}}{\partial t^{2}}=\operatorname{div}_{x} \mathbb{P}^{s}+\rho_{s} \boldsymbol{F}, & \boldsymbol{x} \in \Omega_{s}, t>0, \\
\mathbb{P}^{s}=\alpha_{\eta}\left(\operatorname{div}_{x} \boldsymbol{w}_{s}\right) \cdot \mathbb{I}+\alpha_{\lambda} \mathbb{D}\left(x, \boldsymbol{w}_{s}\right), & \boldsymbol{x} \in \Omega_{s}, t>0 .
\end{aligned}
$$

In (1.1)-(1.6) $\boldsymbol{F}=\boldsymbol{F}(\boldsymbol{x}, t)$ is the given vector of distributed mass forces, $L$ is the characteristic macroscopic size - the diameter of the domain $\Omega^{*}, \tau$ is characteristic duration of physical processes, $\rho_{0}$ is mean density of air under atmosphere pressure, $\rho_{f}$ and $\rho_{s}$ are respectively mean dimensionless densities of liquid and rigid phases, correlated with mean density of air, $g$ is the value of acceleration of gravity, and $p_{0}$ is atmosphere pressure.

Dimensionless constants $\alpha_{i}(i=\tau, \nu, \ldots)$ are defined by the formulas

$$
\left.\begin{array}{c}
\alpha_{\tau}=\frac{L}{g \tau^{2}}, \quad \alpha_{\nu}=\frac{\nu}{\tau L g \rho_{0}}, \quad \alpha_{\mu}=\frac{2 \mu}{\tau L g \rho_{0}}, \\
\alpha_{p}=\frac{c^{2} \rho_{f}}{L g}, \quad \alpha_{\eta}=\frac{\eta}{L g \rho_{0}}, \quad \alpha_{\lambda}=\frac{2 \lambda}{L g \rho_{0}},
\end{array}\right\}
$$


where $\mu$ is the viscosity of fluid or gas, $\nu$ is the bulk viscosity of fluid or gas, $\lambda$ and $\eta$ are elastic Lamé's constants, and $c$ is a speed of sound in fluid.

For the unknown functions $\boldsymbol{w}_{f}, p$, and $\boldsymbol{w}_{s}$, the commonly accepted conditions of continuity of the displacement field and normal tensions are imposed on the interface $\Gamma:=\partial \Omega_{f} \cap \partial \Omega_{s}$ between the two phases (see, for example, [2, 3, 4]):

$$
\begin{aligned}
\mathbb{P}^{f} \cdot \boldsymbol{n}=\mathbb{P}^{s} \cdot \boldsymbol{n}, & \boldsymbol{x} \in \Gamma, t>0, \\
\boldsymbol{w}^{f}=\boldsymbol{w}^{s}, & \boldsymbol{x} \in \Gamma, t>0 .
\end{aligned}
$$

In (1.8) $\boldsymbol{n}$ is the unit normal vector to $\Gamma$. Note that exactly these conditions appear as the result of linearization of the exact nonlinear model.

Finally, system (1.2)-(1.6), (1.8) $-(1.9)$ is endowed by giving a displacement field on $S=\partial \Omega$ and at the moment $t=0$ and by giving a velocity field at the $t=0$. Further without loss of generality, with the aim to simplify the technical outline, we suppose that these boundary conditions are homogeneous.

1.2. Geometry of porous space. In model A a Lipschitz smoothness of the interface between porous space and rigid skeleton is the only restriction on geometry of porous space. In model $\boldsymbol{B}^{\varepsilon}$ the porous medium has geometrically periodic structure. Its formal description is as follows [4, 11].

Firstly a geometric structure inside a pattern unit cell $Y=(0,1)^{3}$ is defined. Let $Y_{s}$ be a 'solid part' of the cell $Y$. The 'liquid part' $Y_{f}$ is its open complement. Set $Y_{s}^{k}:=Y_{s}+\boldsymbol{k}, \boldsymbol{k} \in \mathbb{Z}^{3}$, the translation of $Y_{s}$ on an integervalued vector $\boldsymbol{k}$. Union of such translations along all $\boldsymbol{k}, E_{s}:=\cup_{\boldsymbol{k} \in \mathbb{Z}^{3}} Y_{s}^{k}$ is the 1-periodic repetition of $Y_{s}$ all over $\mathbb{R}^{3}$. Let $E_{f}$ be the open complement of $E_{s}$ in $\mathbb{R}^{3}$. The following assumptions on geometry of $Y_{f}$ and $E_{f}$ are accepted.

(i) $Y_{s}$ is an open connected set of strictly positive measure with a Lipschitz boundary, and $Y_{f}$ also has strictly positive measure on $Y$.

(ii) $E_{f}$ and $E_{s}$ are open sets with $C^{0,1}$-smooth boundaries. The set $E_{f}$ is locally situated on one side of the boundary $\partial E_{f}$, and the set $\mathbf{E}_{s}$ is locally situated on one side of the boundary $\partial E_{s}$ and connected.

Domains $\Omega_{s}^{\varepsilon}$ and $\Omega_{f}^{\varepsilon}$ are intersections of the domain $\Omega$ with the sets $\mathbb{R}_{s}^{3}$ and $\mathbb{R}_{f}^{3}$, where the sets $\mathbb{R}_{s}^{3}$ and $\mathbb{R}_{f}^{3}$ are periodic domains in $\mathbb{R}^{3}$ with generic cells $\varepsilon Y_{s}$ and $\varepsilon Y_{f}$ of the diameter $\varepsilon$, respectively.

Union $\bar{Y}_{s} \cup \bar{Y}_{f}$ is the closed cube $\bar{Y}=\left\{\boldsymbol{y} \in \mathbb{R}^{3}, 0 \leq y_{i} \leq 1, i=1,2,3\right\}$, and the interface $\Gamma^{\varepsilon}=\partial \Omega_{s}^{\varepsilon} \cap \partial \Omega_{f}^{\varepsilon}$ is the $\varepsilon$-periodic repetition of the boundary $\varepsilon \gamma=\varepsilon \partial Y_{f} \cap \partial Y_{s}$ all over $\omega$.

Further by $\bar{\chi}=\chi^{\varepsilon}$ we will denote the characteristic function of the porous space.

For simplicity we accept the following constraint on the domain $\Omega$ and the parameter $\varepsilon$. 
Assumption 1.1. Domain $\Omega$ is cube, $\Omega:=(0,1)^{3}$, and quantity $1 / \varepsilon$ is integer, so that $\Omega$ always contains an integer number of elementary cells $Y_{i}^{\varepsilon}$.

Under this assumption, we have

$$
\bar{\chi}(\boldsymbol{x})=\chi^{\varepsilon}(\boldsymbol{x})=\chi(\boldsymbol{x} / \varepsilon),
$$

where $\chi(\boldsymbol{y})$ is the characteristic function of $Y_{f}$ in $Y$.

We say that a porous space is disconnected (isolated pores) if $\gamma \cap \partial Y=\emptyset$.

1.3. Generalized solutions in models $\mathbf{A}$ and $\boldsymbol{B}^{\varepsilon}$. Define the displacement $\boldsymbol{w}(\boldsymbol{x}, t)$ in the whole domain $\Omega$ by the formula

$$
\boldsymbol{w}(\boldsymbol{x}, t)= \begin{cases}\boldsymbol{w}_{f}(\boldsymbol{x}, t), & \boldsymbol{x} \in \Omega_{f}, \quad t>0, \\ \boldsymbol{w}_{s}(\boldsymbol{x}, t), & \boldsymbol{x} \in \Omega_{s}, \quad t>0,\end{cases}
$$

and the pressures $p(\boldsymbol{x}, t), q(\boldsymbol{x}, t)$, and $\pi(\boldsymbol{x}, t)$ by formulas

$$
p=-\alpha_{p} \bar{\chi} \operatorname{div}_{x} \boldsymbol{w}, \quad \pi=-\alpha_{\eta}(1-\bar{\chi}) \operatorname{div}_{x} \boldsymbol{w}, \quad q=p+\frac{\alpha_{\nu}}{\alpha_{p}} \frac{\partial p}{\partial t} .
$$

Thus introduced new unknown functions should satisfy the system

$$
\begin{gathered}
\alpha_{\tau} \bar{\rho} \frac{\partial^{2} \boldsymbol{w}}{\partial t^{2}}=\operatorname{div}_{x} \mathbb{P}+\bar{\rho} \boldsymbol{F}, \\
\mathbb{P}=\bar{\chi} \alpha_{\mu} \mathbb{D}\left(x, \frac{\partial \boldsymbol{w}}{\partial t}\right)+(1-\bar{\chi}) \alpha_{\lambda} \mathbb{D}(x, \boldsymbol{w})-(q+\pi) \mathbb{I},
\end{gathered}
$$

where $\bar{\rho}=\bar{\chi} \rho_{f}+(1-\bar{\chi}) \rho_{s}$. If $\bar{\chi}(\boldsymbol{x})=\chi^{\varepsilon}(\boldsymbol{x})$, then $\bar{\rho}=\rho^{\varepsilon} \equiv \chi^{\varepsilon} \rho_{f}+\left(1-\chi^{\varepsilon}\right) \rho_{s}$.

Equations (1.13) are understood in the sense of distributions theory. They involve the both equations (1.2) and (1.5) in the domains $\Omega_{f}$ and $\Omega_{s}$, respectively, and the boundary conditions (1.8) and (1.9) on the interface $\Gamma$. There are various equivalent in the sense of distributions forms of representation of equations (1.13). In what follows, it is convenient to write them in the form of the integral equality

$$
\begin{aligned}
& \int_{\Omega_{T}}\left\{\alpha_{\tau} \bar{\rho} \boldsymbol{w} \cdot \frac{\partial^{2} \boldsymbol{\psi}}{\partial t^{2}}-\bar{\rho} \boldsymbol{F} \cdot \boldsymbol{\psi}-\bar{\chi} \alpha_{\mu} \mathbb{D}(x, \boldsymbol{w}): \mathbb{D}\left(x, \frac{\partial \boldsymbol{\psi}}{\partial t}\right)+\right. \\
& \left.\left((1-\bar{\chi}) \alpha_{\lambda} \mathbb{D}(x, \boldsymbol{w})-(q+\pi) \mathbb{I}\right): \mathbb{D}(x, \boldsymbol{\psi})\right\} d \boldsymbol{x} d t=0,
\end{aligned}
$$

where $\boldsymbol{\psi}(\boldsymbol{x}, t)$ is an arbitrary smooth test function, such that $\boldsymbol{\psi}=\partial \boldsymbol{\psi} / \partial t=0$ at the $t=T$ and $\boldsymbol{\psi}=0$ on the boundary $S_{T}$ of the domain $\Omega_{T}, \Omega_{T}=$ $\Omega \times(0, T)$.

In (1.14) by $A: B$ we denote the convolution (or, equivalently, the inner tensor product) of two second-rank tensors along the both indexes, i.e., $A$ : $B=\operatorname{tr}\left(B^{*} \circ A\right)=\sum_{i, j=1}^{3} A_{i j} B_{j i}$. 
Mathematical model (1.12), (1.13b), and (1.14) is endowed by the initial and boundary conditions

$$
\begin{gathered}
\boldsymbol{w}(\boldsymbol{x}, 0)=0, \quad \frac{\partial \boldsymbol{w}}{\partial t}(\boldsymbol{x}, 0)=0, \quad \boldsymbol{x} \in \Omega, \\
\boldsymbol{w}(\boldsymbol{x}, t)=0, \quad \boldsymbol{x} \in S, \quad t>0 .
\end{gathered}
$$

Here the assumption, that the boundary and initial conditions are homogeneous, is not essential.

We will call solution of problem (1.12), (1.13b), (1.14)-(1.16) a generalized solution in model $\boldsymbol{A}$ and, if $\Omega$ is supplemented with periodic structure, a generalized solution in model $\boldsymbol{B}^{\varepsilon}$.

The following assertion states that problem (1.12), (1.13b), (1.14)-(1.16) is well-posed.

Lemma 1.1. Let the interface $\Gamma$ between $\Omega_{s}$ and $\Omega_{f}$ be piece-wise continuously differentiable, parameters $\rho_{f}, \rho_{s}, \alpha_{\tau}, \alpha_{\mu}, \alpha_{\nu}, \alpha_{\lambda}, \alpha_{\eta}$, and $\alpha_{p}$ be strictly positive, and assume that $\boldsymbol{F}, \partial \boldsymbol{F} / \partial t \in L^{2}\left(\Omega_{T}\right)$.

Then, on any finite temporal interval $[0, T]$, problem (1.12), (1.13b), (1.14) -(1.16) has a unique solution $\{\boldsymbol{w}, p, \pi\}$, and this solution admits the bounds

$$
\begin{gathered}
\max _{0<t<T}\left(\sqrt{\alpha_{\eta}}\left\|(1-\bar{\chi}) d i v_{x} \frac{\partial \boldsymbol{w}}{\partial t}(t)\right\|_{2, \Omega}+\sqrt{\alpha_{\lambda}}\left\|(1-\bar{\chi}) \nabla_{x} \frac{\partial \boldsymbol{w}}{\partial t}(t)\right\|_{2, \Omega}\right. \\
\left.+\sqrt{\alpha_{\tau}}\left\|\frac{\partial^{2} \boldsymbol{w}}{\partial t^{2}}(t)\right\|_{2, \Omega}+\sqrt{\alpha_{p}}\left\|\bar{\chi} d i v_{x} \frac{\partial \boldsymbol{w}}{\partial t}(t)\right\|_{2, \Omega}\right)+\sqrt{\alpha_{\nu}}\left\|\bar{\chi} d i v_{x} \frac{\partial^{2} \boldsymbol{w}}{\partial t^{2}}\right\|_{2, \Omega_{T}}+ \\
+\sqrt{\alpha_{\mu}}\left\|\bar{\chi} \nabla_{x} \frac{\partial^{2} \boldsymbol{w}}{\partial t^{2}}\right\|_{2, \Omega_{T}} \leq \frac{C}{\sqrt{\alpha_{\tau}}}\left\||\boldsymbol{F}|+\left|\frac{\partial \boldsymbol{F}}{\partial t}\right|\right\|_{2, \Omega_{T}}
\end{gathered}
$$

where $C$ depends only on $T$.

Due to linearity of the problem, justification of Lemma 1.1 reduces to verification of bounds (1.17). These appear by means of differentiation of Eqs. (1.13) with respect to $t$ (note that $\chi$ and $\bar{\rho}$ do not depend on $t$ ), multiplication of the resulting equation by $\partial^{2} \boldsymbol{w} / \partial t^{2}$, and integration by parts. Pressures $q$ and $\pi$ are estimated directly from Eqs. (1.12).

Further the focus of this article is centered solely on model $\boldsymbol{B}^{\varepsilon}$, in which coefficients of Eqs. (1.12) and (1.13) depend continuously on the small parameter $\varepsilon, \bar{\rho}=\rho^{\varepsilon}(x)=\chi^{\varepsilon} \rho_{f}+\left(1-\chi^{\varepsilon}\right) \rho_{s}$ and $\left\{\boldsymbol{w}^{\varepsilon}, p^{\varepsilon}, q^{\varepsilon}, \pi^{\varepsilon}\right\}$ is a corresponding generalized solution. We aim to find out the limiting regimes of the model as $\varepsilon \searrow 0$.

\section{$\S 2$. Formulation of the main results}


Suppose additionally that there exist limits (finite or infinite)

$$
\begin{aligned}
& \lim _{\varepsilon \searrow 0} \alpha_{\nu}(\varepsilon)=\nu_{0}, \quad \lim _{\varepsilon \searrow 0} \alpha_{p}(\varepsilon)=p_{*}, \quad \lim _{\varepsilon \searrow 0} \alpha_{\eta}(\varepsilon)=\eta_{0} . \\
& \lim _{\varepsilon \searrow 0} \frac{\alpha_{\mu}}{\varepsilon^{2}}=\mu_{1}, \quad \lim _{\varepsilon \searrow 0} \frac{\varepsilon^{2} \alpha_{p}}{\alpha_{\mu}}=p_{1}, \quad \lim _{\varepsilon \searrow 0} \frac{\alpha_{\lambda} \varepsilon^{2}}{\alpha_{\mu}}=\lambda_{1}, \\
& \lim _{\varepsilon \searrow 0} \frac{\alpha_{\eta} \varepsilon^{2}}{\alpha_{\mu}}=\eta_{1}, \quad, \lim _{\varepsilon \searrow 0} \frac{\alpha_{\eta}}{\alpha_{\lambda}}=\eta_{2}, \quad \lim _{\varepsilon \searrow 0} \frac{\alpha_{p}}{\alpha_{\lambda}}=p_{2} .
\end{aligned}
$$

In what follows we assume that

Assumption 2.1. Dimensionless parameters in the model $\boldsymbol{B}^{\varepsilon}$ satisfy restrictions

$$
p_{*}^{-1}, \quad \mu_{0}, \quad \nu_{0}, \quad \lambda_{0}^{-1}<\infty ; \quad 0<\tau_{0}+\mu_{1} .
$$

All parameters may take all permitted values. For example, if $\tau_{0}=0$ or $p_{*}^{-1}=0$, then all terms in final equations containing these parameters disappear.

The following Theorems 2.1 2.4 are the main results of the paper.

Theorem 2.1. Assume that conditions of Lemma 1.1 hold and that $\left\{\boldsymbol{w}^{\varepsilon}, q^{\varepsilon}, p^{\varepsilon}, \pi^{\varepsilon}\right\}$ is a generalized solution in model $\boldsymbol{B}^{\varepsilon}$.

The following assertions are valid:

(I) If

$$
\lambda_{0}<\infty
$$

then

$$
\begin{gathered}
\max _{0 \leq t \leq T}||\left|\boldsymbol{w}^{\varepsilon}(t)\right|+\sqrt{\alpha_{\mu}} \chi^{\varepsilon}\left|\nabla_{x} \boldsymbol{w}^{\varepsilon}(t)\right|+\left(1-\chi^{\varepsilon}\right)\left|\nabla_{x} \boldsymbol{w}^{\varepsilon}(t)\right| \|_{2, \Omega} \leq I_{F}, \\
\left\|q^{\varepsilon}\right\|_{2, \Omega_{T}}+\left\|p^{\varepsilon}\right\|_{2, \Omega_{T}}+\left\|\pi^{\varepsilon}\right\|_{2, \Omega_{T}} \leq I_{F},
\end{gathered}
$$

where $I_{F}=C\||\boldsymbol{F}|+|\partial \boldsymbol{F} / \partial t|\|_{2, \Omega_{T}}$ and $C$ is a constant independent of $\varepsilon$.

(II) If

$$
\lambda_{0}=\infty, \quad \mu_{1}=\infty, \quad 0<\lambda_{1}<\infty,
$$

then estimates (2.2), (2.3) hold for re-normalized displacements

$$
w^{\varepsilon} \rightarrow \varepsilon^{-2} \alpha_{\mu} w^{\varepsilon}
$$

with re-normalized parameters

$$
\alpha_{\mu} \rightarrow \varepsilon^{2}, \quad \alpha_{\lambda} \rightarrow \varepsilon^{2} \frac{\alpha_{\lambda}}{\alpha_{\mu}}, \quad \alpha_{\tau} \rightarrow \varepsilon^{2} \frac{\alpha_{\tau}}{\alpha_{\mu}}, \quad \alpha_{\nu} \rightarrow \varepsilon^{2} \frac{\alpha_{\nu}}{\alpha_{\mu}}, \quad \alpha_{p} \rightarrow \varepsilon^{2} \frac{\alpha_{p}}{\alpha_{\mu}} .
$$


(III) If

$$
\lambda_{0}=\infty, \quad \mu_{1}<\infty,
$$

then for displacements $\boldsymbol{w}^{\varepsilon}$ hold true estimates (2.2) and under condition

$$
p_{*}<\infty,
$$

for the pressures $q^{\varepsilon}$ and $p^{\varepsilon}$ in the liquid component hold true estimates (2.3).

If instead of restriction (2.4) hold true conditions

$$
0<p_{2} ; \quad \boldsymbol{F}=\nabla \Phi, \quad \frac{\partial \Phi}{\partial t}, \quad\left|\frac{\partial \boldsymbol{F}}{\partial t}\right| \in L^{2}\left(\Omega_{T}\right),
$$

then

$$
\max _{0 \leq t \leq T}\left(\left\|\left(1-\chi^{\varepsilon}\right) \nabla_{x}\left(\alpha_{\lambda} \boldsymbol{w}^{\varepsilon}(t)\right)\right\|_{2, \Omega}+\left\|\chi^{\varepsilon} \operatorname{div}_{x}\left(\alpha_{\lambda} \boldsymbol{w}^{\varepsilon}(t)\right)\right\|_{2, \Omega} \leq I_{F}^{(1)},\right.
$$

where $I_{F}^{(1)}=C\||| \boldsymbol{F}|+| \partial \Phi / \partial t|+| \partial \boldsymbol{F} / \partial t \mid\|_{2, \Omega_{T}}$ and $C$ is a constant independent of $\varepsilon$.

These last estimates imply (2.3).

Note, that for the last case $\left\{\lambda_{0}=\infty, \mu_{1}<\infty\right\}$ we can get same estimates (2.2), (2.3) and (2.6), if instead restrictions (2.5) we consider

\section{Assumption 2.2.}

$$
\boldsymbol{F}=\boldsymbol{F}^{\varepsilon}(\boldsymbol{x}, t)\left(1-\chi^{\varepsilon}\right)
$$

and sequences $\left\{\boldsymbol{F}^{\varepsilon}\right\}$ and $\left\{\partial \boldsymbol{F}^{\varepsilon} / \partial t\right\}$ are uniformly bounded with respect to $\varepsilon$ in $L^{2}\left(\Omega_{T}\right)$.

Theorem 2.2. Assume that the hypotheses in Theorem 2.1 hold, and

$$
\lambda_{0}<\infty, \quad \mu_{0}=0 .
$$

Then functions $\boldsymbol{w}^{\varepsilon}$ admit an extension $\boldsymbol{u}^{\varepsilon}$ from $\Omega_{s, T}^{\varepsilon}=\Omega_{s}^{\varepsilon} \times(0, T)$ into $\Omega_{T}$ such that the sequence $\left\{\boldsymbol{u}^{\varepsilon}\right\}$ converges strongly in $L^{2}\left(\Omega_{T}\right)$ and weakly in $L^{2}\left((0, T) ; W_{2}^{1}(\Omega)\right)$ to the functions $\boldsymbol{u}$. At the same time, sequences $\left\{\boldsymbol{w}^{\varepsilon}\right\}$, $\left\{p^{\varepsilon}\right\},\left\{q^{\varepsilon}\right\}$, and $\left\{\pi^{\varepsilon}\right\}$ converge weakly in $L^{2}\left(\Omega_{T}\right)$ to $\boldsymbol{w}, p$, q, and $\pi$, respectively.

The following assertions for these limiting functions hold true:

(I) If $\mu_{1}=\infty$ or the porous space is disconnected (a case of isolated pores), then $\boldsymbol{w}=\boldsymbol{u}$ and the functions $\boldsymbol{u}, p, q$, and $\pi$ satisfy in the domain $\Omega_{T}$ the following initial-boundary value problem:

$$
\tau_{0} \hat{\rho} \frac{\partial^{2} \boldsymbol{u}}{\partial t^{2}}=\operatorname{div}_{x}\left\{\lambda_{0} \mathbb{A}_{0}^{s}: \mathbb{D}(x, \boldsymbol{u})+B_{0}^{s} d i v_{x} \boldsymbol{u}+B_{1}^{s} q-(q+\pi) \cdot \mathbb{I}\right\}+\hat{\rho} \boldsymbol{F},
$$




$$
\begin{gathered}
\frac{1}{\eta_{0}} \pi+C_{0}^{s}: \mathbb{D}(x, \boldsymbol{u})+a_{0}^{s} \operatorname{div}_{x} \boldsymbol{u}+a_{1}^{s} q=0, \\
\frac{1}{p_{*}} p+\frac{1}{\eta_{0}} \pi+\operatorname{div}_{x} \boldsymbol{u}=0, \quad p+\nu_{0} p_{*}^{-1} \frac{\partial p}{\partial t}=q,
\end{gathered}
$$

where $\hat{\rho}=m \rho_{f}+(1-m) \rho_{s}, m=\langle\chi\rangle$. The symmetric strictly positively defined constant fourth-rank tensor $\mathbb{A}_{0}^{s}$, matrices $C_{0}^{s}, B_{0}^{s}$ and $B_{1}^{s}$ and constants $a_{0}^{s}$ and $a_{1}^{s}$ are defined below by formulas (5.30), (5.32) - (5.34).

Differential equations (2.7)-(2.8) are endowed with the homogeneous initial and boundary conditions

$$
\left.\tau_{0} \boldsymbol{u}\right|_{t=0}=\left.\tau_{0} \frac{\partial \boldsymbol{u}}{\partial t}\right|_{t=0}=0, \quad \boldsymbol{x} \in \Omega, \quad \boldsymbol{u}(\boldsymbol{x}, t)=0, \quad \boldsymbol{x} \in S .
$$

(II) If $\mu_{1}<\infty$, then the weak limits $\boldsymbol{u}, \boldsymbol{w}^{f}, p, q, \pi$ of sequences $\left\{\boldsymbol{u}^{\varepsilon}\right\}$, $\left\{\chi^{\varepsilon} \boldsymbol{w}^{\varepsilon}\right\},\left\{p^{\varepsilon}\right\},\left\{q^{\varepsilon}\right\},\left\{\pi^{\varepsilon}\right\}$ satisfy the initial-boundary value problem consisting of the balance of momentum equation

$$
\begin{aligned}
& \tau_{0} \rho_{f} \frac{\partial \boldsymbol{v}}{\partial t}+\tau_{0} \rho_{s}(1-m) \frac{\partial^{2} \boldsymbol{u}}{\partial t^{2}}+\nabla(q+\pi)-\hat{\rho} \boldsymbol{F}= \\
& \operatorname{div}_{x}\left\{\lambda_{0} \mathbb{A}_{0}^{s}: \mathbb{D}(x, \boldsymbol{u})+B_{0}^{s} d i v_{x} \boldsymbol{u}+B_{1}^{s} q\right\},
\end{aligned}
$$

where $\boldsymbol{v}=\partial \boldsymbol{w}^{f} / \partial t$ and $\mathbb{A}_{0}^{s}, B_{0}^{s}$ and $B_{1}^{s}$ are the same as in (2.7), the continuity equations (2.8), the continuity equation and the state equation

$$
\frac{1}{p_{*}} p+\frac{1}{\eta_{0}} \pi+d i v_{x} \boldsymbol{w}^{f}=(m-1) d i v_{x} \boldsymbol{u}, \quad p+\nu_{0} p_{*}^{-1} \frac{\partial p}{\partial t}=q,
$$

and Darcy's law in the form

$$
\boldsymbol{v}=m \frac{\partial \boldsymbol{u}}{\partial t}+\int_{0}^{t} B_{1}\left(\mu_{1}, t-\tau\right) \cdot\left(-\nabla_{x} q+\rho_{f} \boldsymbol{F}-\tau_{0} \rho_{f} \frac{\partial^{2} \boldsymbol{u}}{\partial \tau^{2}}\right)(\boldsymbol{x}, \tau) d \tau
$$

in the case $\tau_{0}>0$ and $\mu_{1}>0$, Darcy's law in the form

$$
\boldsymbol{v}=m \frac{\partial \boldsymbol{u}}{\partial t}+B_{2}\left(\mu_{1}\right) \cdot\left(-\nabla q+\rho_{f} \boldsymbol{F}\right),
$$

in the case $\tau_{0}=0$ and $\mu_{1}>0$, and, finally, Darcy's law in the form

$$
\boldsymbol{v}=B_{3} \cdot \frac{\partial \boldsymbol{u}}{\partial t}+\frac{1}{\tau_{0} \rho_{f}}\left(m \mathbb{I}-B_{3}\right) \cdot \int_{0}^{t}\left(-\nabla q(\boldsymbol{x}, \tau)+\rho_{f} \boldsymbol{F}(\boldsymbol{x}, \tau)\right) d \tau,
$$

in the case $\tau_{0}>0$ and $\mu_{1}=0$.

This problem is endowed with initial and boundary conditions (2.10) and the boundary condition

$$
\boldsymbol{v}(\boldsymbol{x}, t) \cdot n(\boldsymbol{x})=0, \quad \boldsymbol{x} \in S, \quad t>0,
$$

for the velocity $\boldsymbol{v}$ of the fluid component.

In (2.13)-(2.16) $n(\boldsymbol{x})$ is the unit normal vector to $S$ at a point $\boldsymbol{x} \in S$, and matrices $B_{1}\left(\mu_{1}, t\right), B_{2}\left(\mu_{1}\right)$ and $\left(m \mathbb{I}-B_{3}\right)$ are defined below by formulas (5.42), (5.44) and (5.46). 
Theorem 2.3. Assume that the hypotheses in Theorem 2.1 hold, and that

$$
\lambda_{0}=\infty
$$

(I) If $\mu_{1}<\infty$ and one of conditions (2.4) or (2.5) holds true, then sequences $\left\{\chi^{\varepsilon} \boldsymbol{w}^{\varepsilon}\right\},\left\{p^{\varepsilon}\right\}$ and $\left\{q^{\varepsilon}\right\}$ converge weakly in $L^{2}\left(\Omega_{T}\right)$ to $\boldsymbol{w}^{f}, p$, and $q$ respectively. The functions $\boldsymbol{w}^{\varepsilon}$ admit an extension $\boldsymbol{u}^{\varepsilon}$ from $\Omega_{s}^{\varepsilon} \times(0, T)$ into $\Omega_{T}$ such that the sequence $\left\{\boldsymbol{u}^{\varepsilon}\right\}$ converges strongly in $L^{2}\left(\Omega_{T}\right)$ and weakly in $L^{2}\left((0, T) ; W_{2}^{1}(\Omega)\right)$ to zero and

1) if $\tau_{0}>0$ and $\mu_{1}>0$, then functions $\boldsymbol{v}=\partial \boldsymbol{w}^{f} / \partial t, p$ and $q$ solve in the domain $\Omega_{T}$ the problem $\left(F_{1}\right)$, where

$$
\begin{gathered}
\boldsymbol{v}=\int_{0}^{t} B_{1}\left(\mu_{1}, t-\tau\right) \cdot\left(-\nabla q(\boldsymbol{x}, \tau)+\rho_{f} \boldsymbol{F}(\boldsymbol{x}, \tau)\right) d \tau \\
p+\nu_{0} p_{*}^{-1} \frac{\partial p}{\partial t}=q, \quad \frac{1}{p_{*}} \frac{\partial p}{\partial t}+\operatorname{div}_{x} \boldsymbol{v}=0
\end{gathered}
$$

2) if $\tau_{0}=0$ and $\mu_{1}>0$, then functions $\boldsymbol{v}, p$ and $q$ solve in the domain $\Omega_{T}$ the problem $\left(F_{2}\right)$, where $\boldsymbol{v}$ satisfies Darcy's law in the form

$$
\boldsymbol{v}=B_{2}\left(\mu_{1}\right) \cdot\left(-\nabla q+\rho_{f} \boldsymbol{F}\right),
$$

and pressures $p$ and $q$ satisfy equations (2.18);

finally,

3) if $\tau_{0}>0$ and $\mu_{1}=0$, then functions $\boldsymbol{v}, p$ and $q$ solve in the domain $\Omega_{T}$ the problem $\left(F_{3}\right)$, where $\boldsymbol{v}$ satisfies Darcy's law in the form

$$
\boldsymbol{v}=\frac{1}{\tau_{0} \rho_{f}}\left(m \mathbb{I}-B_{3}\right) \cdot \int_{0}^{t}\left(-\nabla q(\boldsymbol{x}, \tau)+\rho_{f} \boldsymbol{F}(\boldsymbol{x}, \tau)\right) d \tau,
$$

and pressures $p$ and $q$ satisfy equations (2.18).

Problems $F_{1}-F_{3}$ are endowed with boundary condition (2.16).

(II) If $\mu_{1}<\infty$ and conditions (2.5) hold true, then the sequence $\left\{\alpha_{\lambda} \boldsymbol{u}^{\varepsilon}\right\}$ converges strongly in $L^{2}\left(\Omega_{T}\right)$ and weakly in $L^{2}\left((0, T) ; W_{2}^{1}(\Omega)\right)$ to function $\boldsymbol{u}$ and the sequence $\left\{\pi^{\varepsilon}\right\}$ converges weakly in $L^{2}\left(\Omega_{T}\right)$ to the function $\pi$. The limiting functions $\boldsymbol{u}$ and $\pi$ satisfy the boundary value problem in the domain $\Omega$

$$
\begin{gathered}
0=\operatorname{div}_{x}\left\{\mathbb{A}_{0}^{s}: \mathbb{D}(x, \boldsymbol{u})+B_{0}^{s} \operatorname{div}_{x} \boldsymbol{u}+B_{1}^{s} q-(q+\pi) \cdot \mathbb{I}\right\}+\hat{\rho} \boldsymbol{F}, \\
\frac{1}{\eta_{2}} \pi+C_{0}^{s}: \mathbb{D}(x, \boldsymbol{u})+a_{0}^{s} d i v_{x} \boldsymbol{u}+a_{1}^{s} q=0,
\end{gathered}
$$

where the function $q$ is referred to as given. It is defined from the corresponding of Problems $F_{1}-F_{3}$ (the choice of the problem depends on $\tau_{0}$ and $\mu_{1}$ ). The 
symmetric strictly positively defined constant fourth-rank tensor $\mathbb{A}_{0}^{s}$, matrices $C_{0}^{s}, B_{0}^{s}$ and $B_{1}^{s}$ and constants $a_{0}^{s}$ and $a_{1}^{s}$ are defined below by formulas (5.30), (5.32) - (5.33), in which we have $\eta_{0}=\eta_{2}$ and $\lambda_{0}=1$.

This problem is endowed with the homogeneous boundary conditions.

(III) If $\mu_{1}=\infty, p_{1}^{-1}, \eta_{1}^{-1}<\infty$ and $0<\lambda_{1}<\infty$, then there exist weak limits $\boldsymbol{w}^{f}, p$ and $\pi$ of the sequences $\left\{\alpha_{\mu} \varepsilon^{-2} \chi^{\varepsilon} \boldsymbol{w}^{\varepsilon}\right\},\left\{p^{\varepsilon}\right\}$ and $\left\{\pi^{\varepsilon}\right\}$ and a strong limit $\boldsymbol{u}$ of the sequence $\left\{\alpha_{\mu} \varepsilon^{-2} \boldsymbol{u}^{\varepsilon}\right\}$ in $L^{2}\left(\Omega_{T}\right)$, which satisfy in $\Omega_{T}$ the following initial boundary-value problem:

$$
\left.\begin{array}{r}
d i v_{x}\left\{\lambda_{1} \mathbb{A}_{0}^{s}: \mathbb{D}(x, \boldsymbol{u})+B_{0}^{s} d i v_{x} \boldsymbol{u}+B_{1}^{s} p-(p+\pi) \cdot \mathbb{I}\right\}+\hat{\rho} \boldsymbol{F}=0, \\
\frac{\partial \boldsymbol{w}^{f}}{\partial t}=\frac{\partial \boldsymbol{u}}{\partial t}+B_{2}(1) \cdot\left(-\nabla p+\rho_{f} \boldsymbol{F}\right), \\
\frac{1}{p_{1}} p+\frac{1}{\eta_{1}} \pi+\operatorname{div}_{x} \boldsymbol{w}^{f}=(m-1) d i v_{x} \boldsymbol{u}, \\
\frac{1}{\eta_{1}} \pi+C_{0}^{s}: \mathbb{D}(x, \boldsymbol{u})+a_{0}^{s} d i v_{x} \boldsymbol{u}+a_{1}^{s} p=0 .
\end{array}\right\}
$$

Here the symmetric strictly positively defined constant fourth-rank tensor $\mathbb{A}_{0}^{s}$, matrices $C_{0}^{s}, B_{0}^{s}$ and $B_{1}^{s}$ and constants $a_{0}^{s}$ and $a_{1}^{s}$ are defined below by formulas (5.30), (5.32) - (5.34), in which we have $\eta_{0}=\eta_{1}$ and $\lambda_{0}=\lambda_{1}$.

This problem is endowed with the homogeneous initial and boundary conditions.

$(\boldsymbol{I} \boldsymbol{V})$ If $\mu_{1}=\infty$ and $\lambda_{1}=\infty$, then the corresponding problem for displacements $\left\{\alpha_{\mu} \varepsilon^{-2} \boldsymbol{w}^{\varepsilon}\right\}$ has been considered in parts $(\boldsymbol{I})-(\boldsymbol{I I})$ of the present theorem.

Theorem 2.4. Assume that the hypotheses in Theorem 2.1 hold, and that

$$
0<\mu_{0}, \quad \lambda_{0}<\infty
$$

Then weak limits $\boldsymbol{w}, p, q$ and $\pi$ of sequences $\left\{\boldsymbol{w}^{\varepsilon}\right\},\left\{p^{\varepsilon}\right\},\left\{\pi^{\varepsilon}\right\}$ and $\left\{q^{\varepsilon}\right\}$ satisfy in $\Omega_{T}$ the following initial-boundary value problem:

$$
\left.\begin{array}{r}
\tau_{0} \hat{\rho} \frac{\partial^{2} \boldsymbol{w}}{\partial t^{2}}+\nabla(q+\pi)-\hat{\rho} \boldsymbol{F}= \\
\operatorname{div}_{x}\left(\mathbb{A}_{2}: \mathbb{D}\left(x, \frac{\partial \boldsymbol{w}}{\partial t}\right)+\mathbb{A}_{3}: \mathbb{D}(x, \boldsymbol{w})+B_{4} d i v_{x} \boldsymbol{w}+\right. \\
\left.\int_{0}^{t}\left(\mathbb{A}_{4}(t-\tau): \mathbb{D}(x, \boldsymbol{w}(\boldsymbol{x}, \tau))+B_{5}(t-\tau) d i v_{x} \boldsymbol{w}(\boldsymbol{x}, \tau)\right) d \tau\right), \\
\frac{1}{p_{*}} p+m d i v_{x} \boldsymbol{w}= \\
-\int_{0}^{t}\left(C_{2}(t-\tau): \mathbb{D}(x, \boldsymbol{w}(\boldsymbol{x}, \tau))+a_{2}(t-\tau) d i v_{x} \boldsymbol{w}(\boldsymbol{x}, \tau)\right) d \tau .
\end{array}\right\}
$$




$$
\begin{array}{r}
\frac{1}{\eta_{0}} \pi+(1-m) d i v_{x} \boldsymbol{w}= \\
-\int_{0}^{t}\left(C_{3}(t-\tau): \mathbb{D}(x, \boldsymbol{w}(\boldsymbol{x}, \tau))+a_{3}(t-\tau) d i v_{x} \boldsymbol{w}(\boldsymbol{x}, \tau)\right) d \tau, \\
q=p+\frac{\nu_{0}}{p_{*}} \frac{\partial p}{\partial t} .
\end{array}
$$

Here $\mathbb{A}_{2}, \mathbb{A}_{2}$ and $\mathbb{A}_{4}$ are fourth-rank tensors, $B_{4}, B_{5}, C_{2}$ and $C_{3}$ are matrices and $a_{2}$ and $a_{3}$ are scalars. The exact expressions for these objects are given below by formulas (7.26) -(7.31).

The problem is supplemented by the homogeneous initial and boundary conditions. (see in Eqs. (2.10) in Theorem 2.2).

If the porous space is connected then $\mathbb{A}_{2}$ is strictly positively defined symmetric tensor.

If the porous space is disconnected, which is a case of isolated pores, then $\mathbb{A}_{2}=0$ and the system (2.24) degenerates into nonlocal anisotropic Lam'e's system with strictly positively defined and symmetric tensor $\mathbb{A}_{3}$.

\section{§3. Preliminaries}

3.1. Two-scale convergence. Justification of Theorems 2.12 .4 relies on systematic use of the method of two-scale convergence, which had been proposed by G. Nguetseng [8] and has been applied recently to a wide range of homogenization problems (see, for example, the survey [10]).

Definition 3.1. A sequence $\left\{\varphi^{\varepsilon}\right\} \subset L^{2}\left(\Omega_{T}\right)$ is said to be two-scale convergent to a limit $\varphi \in L^{2}\left(\Omega_{T} \times Y\right)$ if and only if for any 1-periodic in $\boldsymbol{y}$ function $\sigma=\sigma(\boldsymbol{x}, t, \boldsymbol{y})$ the limiting relation

$$
\lim _{\varepsilon \searrow 0} \int_{\Omega_{T}} \varphi^{\varepsilon}(\boldsymbol{x}, t) \sigma(\boldsymbol{x}, t, \boldsymbol{x} / \varepsilon) d \boldsymbol{x} d t=\int_{\Omega_{T}} \int_{Y} \varphi(\boldsymbol{x}, t, \boldsymbol{y}) \sigma(\boldsymbol{x}, t, \boldsymbol{y}) d \boldsymbol{y} d \boldsymbol{x} d t
$$

holds.

Existence and main properties of weakly convergent sequences are established by the following fundamental theorem [8, 10]:

\section{Theorem 3.1. (Nguetseng's theorem)}

1. Any bounded in $L^{2}(Q)$ sequence contains a subsequence, two-scale convergent to some limit $\varphi \in L^{2}\left(\Omega_{T} \times Y\right)$.

2. Let sequences $\left\{\varphi^{\varepsilon}\right\}$ and $\left\{\varepsilon \nabla_{x} \varphi^{\varepsilon}\right\}$ be uniformly bounded in $L^{2}\left(\Omega_{T}\right)$. Then there exist a 1-periodic in $\boldsymbol{y}$ function $\varphi=\varphi(\boldsymbol{x}, t, \boldsymbol{y})$ and a subsequence $\left\{\varphi^{\varepsilon}\right\}$ such that $\varphi, \nabla_{y} \varphi \in L^{2}\left(\Omega_{T} \times Y\right)$, and $\varphi^{\varepsilon}$ and $\varepsilon \nabla_{x} \varphi^{\varepsilon}$ two-scale converge to $\varphi$ and $\nabla_{y} \varphi$, respectively.

3. Let sequences $\left\{\varphi^{\varepsilon}\right\}$ and $\left\{\nabla_{x} \varphi^{\varepsilon}\right\}$ be bounded in $L^{2}(Q)$. Then there exist 
functions $\varphi \in L^{2}\left(\Omega_{T}\right)$ and $\psi \in L^{2}\left(\Omega_{T} \times Y\right)$ and a subsequence from $\left\{\varphi^{\varepsilon}\right\}$ such that $\psi$ is 1-periodic in $\boldsymbol{y}, \nabla_{y} \psi \in L^{2}\left(\Omega_{T} \times Y\right)$, and $\varphi^{\varepsilon}$ and $\nabla_{x} \varphi^{\varepsilon}$ two-scale converge to $\varphi$ and $\nabla_{x} \varphi(\boldsymbol{x}, t)+\nabla_{y} \psi(\boldsymbol{x}, t, \boldsymbol{y})$, respectively.

Corollary 3.1. Let $\sigma \in L^{2}(Y)$ and $\sigma^{\varepsilon}(\boldsymbol{x}):=\sigma(\boldsymbol{x} / \varepsilon)$. Assume that a sequence $\left\{\varphi^{\varepsilon}\right\} \subset L^{2}\left(\Omega_{T}\right)$ two-scale converges to $\varphi \in L^{2}\left(\Omega_{T} \times Y\right)$. Then the sequence $\sigma^{\varepsilon} \varphi^{\varepsilon}$ two-scale converges to $\sigma \varphi$.

3.2. An extension lemma. The typical difficulty in homogenization problems while passing to a limit in Model $B^{\varepsilon}$ as $\varepsilon \searrow 0$ arises because of the fact that the bounds on the gradient of displacement $\nabla_{x} \boldsymbol{w}^{\varepsilon}$ may be distinct in liquid and rigid phases. The classical approach in overcoming this difficulty consists of constructing of extension to the whole $\Omega$ of the displacement field defined merely on $\Omega_{s}$. The following lemma is valid due to the well-known results from [12, 13]. We formulate it in appropriate for us form:

Lemma 3.1. Suppose that assumptions of Sec. 1.2 on geometry of periodic structure hold, $\psi^{\varepsilon} \in W_{2}^{1}\left(\Omega_{s}^{\varepsilon}\right)$ and $\psi^{\varepsilon}=0$ on $S_{s}^{\varepsilon}=\partial \Omega_{s}^{\varepsilon} \cap \partial \Omega$ in the trace sense. Then there exists a function $\sigma^{\varepsilon} \in W_{2}^{1}(\Omega)$ such that its restriction on the sub-domain $\Omega_{s}^{\varepsilon}$ coincide with $\psi^{\varepsilon}$, i.e.,

$$
\left(1-\chi^{\varepsilon}(\boldsymbol{x})\right)\left(\sigma^{\varepsilon}(\boldsymbol{x})-\psi^{\varepsilon}(\boldsymbol{x})\right)=0, \quad \boldsymbol{x} \in \Omega,
$$

and, moreover, the estimate

$$
\left\|\sigma^{\varepsilon}\right\|_{2, \Omega} \leq C\left\|\psi^{\varepsilon}\right\|_{2, \Omega_{s}^{\varepsilon}}, \quad\left\|\nabla_{x} \sigma^{\varepsilon}\right\|_{2, \Omega} \leq C\left\|\nabla_{x} \psi^{\varepsilon}\right\|_{2, \Omega_{s}^{\varepsilon}}
$$

hold true, where the constant $C$ depends only on geometry $Y$ and does not depend on $\varepsilon$.

3.3. Friedrichs-Poincaré's inequality in periodic structure. The following lemma was proved by L. Tartar in [3, Appendix]. It specifies Friedrichs-Poincaré's inequality for $\varepsilon$-periodic structure.

Lemma 3.2. Suppose that assumptions on the geometry of $\Omega_{f}^{\varepsilon}$ hold true. Then for any function $\varphi \in \stackrel{\circ}{W_{2}^{1}}\left(\Omega_{f}^{\varepsilon}\right)$ the inequality

$$
\int_{\Omega_{f}^{\varepsilon}}|\varphi|^{2} d \boldsymbol{x} \leq C \varepsilon^{2} \int_{\Omega_{f}^{\varepsilon}}\left|\nabla_{x} \varphi\right|^{2} d \boldsymbol{x}
$$

holds true with some constant $C$, independent of $\varepsilon$.

3.4. Some notation. Further we denote

1)

$$
\langle\Phi\rangle_{Y}=\int_{Y} \Phi d y, \quad\langle\Phi\rangle_{Y_{f}}=\int_{Y} \chi \Phi d y, \quad\langle\Phi\rangle_{Y_{s}}=\int_{Y}(1-\chi) \Phi d y,
$$




$$
\langle\varphi\rangle_{\Omega}=\int_{\Omega} \varphi d x, \quad\langle\varphi\rangle_{\Omega_{T}}=\int_{\Omega_{T}} \varphi d x d t
$$

2) If $\mathbf{a}$ and $\mathbf{b}$ are two vectors then the matrix $\mathbf{a} \otimes \mathbf{b}$ is defined by the formula

$$
(\mathbf{a} \otimes \mathbf{b}) \cdot \mathbf{c}=\mathbf{a}(\mathbf{b} \cdot \mathbf{c})
$$

for any vector $\mathbf{c}$.

3) If $B$ and $C$ are two matrices, then $B \otimes C$ is a forth-rank tensor such that its convolution with any matrix $A$ is defined by the formula

$$
(B \otimes C): A=B(C: A)
$$

. 4) By $\mathbb{I}^{i j}$ we denote the $3 \times 3$-matrix with just one non-vanishing entry, which is equal to one and stands in the $i$-th row and the $j$-th column.

5) We also introduce

$$
J^{i j}=\frac{1}{2}\left(\mathbb{I}^{i j}+\mathbb{I}^{j i}\right)=\frac{1}{2}\left(\boldsymbol{e}_{i} \otimes \boldsymbol{e}_{j}+\boldsymbol{e}_{j} \otimes \boldsymbol{e}_{i}\right),
$$

where $\left(\boldsymbol{e}_{1}, \boldsymbol{e}_{2}, \boldsymbol{e}_{3}\right)$ are the standard Cartesian basis vectors.

\section{$\S 4$. Proof of Theorem 2.1}

4.1. Let $\lambda_{0}<\infty$.

If restriction $\tau_{0}>0$ holds, then (2.2) follow from the lemma 1.1.

Let $p_{*}<\infty$ and $\eta_{0}<\infty$. Then pressures $p^{\varepsilon}$ and $\pi^{\varepsilon}$ are bounded from equations (1.12) with the help of (1.17). The pressure $q^{\varepsilon}$ is bounded from

$$
q^{\varepsilon}=p^{\varepsilon}-\alpha_{\nu} \chi^{\varepsilon} \operatorname{div}_{x} \frac{\partial \boldsymbol{w}^{\varepsilon}}{\partial t}
$$

with the help of (1.17).

If $p_{*}=\infty$, then estimate (2.3) for the sum of pressures $\left(q^{\varepsilon}+\pi^{\varepsilon}\right)$ follows from the basic integral identity (1.14) and estimates (1.17) as an estimate for the corresponding functional, if we re-normalize the pressures $\left(q^{\varepsilon}+\pi^{\varepsilon}\right)$ such that

$$
\int_{\Omega}\left(q^{\varepsilon}(\boldsymbol{x}, t)+\pi^{\varepsilon}(\boldsymbol{x}, t)\right) d \boldsymbol{x}=0 .
$$

. Indeed, the basic integral identity (1.14) and estimates (1.17) imply

$$
\left|\int_{\Omega}\left(q^{\varepsilon}+\pi^{\varepsilon}\right) \operatorname{div}_{x} \boldsymbol{\psi} d \boldsymbol{x}\right| \leq C\|\nabla \boldsymbol{\psi}\|_{2, \Omega}
$$

Choosing now $\boldsymbol{\psi}$ such that $\left(q^{\varepsilon}+\pi^{\varepsilon}\right)=\operatorname{div}_{x} \boldsymbol{\psi}$ we get the desired estimate for the sum of pressures $\left(q^{\varepsilon}+\pi^{\varepsilon}\right)$. Such a choice is always possible (see [14]), if we put

$\boldsymbol{\psi}=\nabla \varphi+\boldsymbol{\psi}_{\mathbf{0}}, \quad \operatorname{div}_{x} \boldsymbol{\psi}_{\mathbf{0}}=0, \quad \Delta \varphi=q^{\varepsilon}+\pi^{\varepsilon},\left.\quad \varphi\right|_{\partial \Omega}=0,\left.\quad\left(\nabla \varphi+\boldsymbol{\psi}_{\mathbf{0}}\right)\right|_{\partial \Omega}=0$. 
Note that the re-normalization of the pressures $\left(q^{\varepsilon}+\pi^{\varepsilon}\right)$ transforms continuity and state equations (1.12) for pressures into

$$
\begin{gathered}
\frac{1}{\alpha_{p}} p^{\varepsilon}+\chi^{\varepsilon} \operatorname{div}_{x} \boldsymbol{w}^{\varepsilon}=-\frac{1}{m} \beta^{\varepsilon} \chi^{\varepsilon} \\
\frac{1}{\alpha_{\eta}} \pi^{\varepsilon}+\left(1-\chi^{\varepsilon}\right) \operatorname{div}_{x} \boldsymbol{w}^{\varepsilon}=\left(1-\chi^{\varepsilon}\right) \gamma_{s}^{\varepsilon} \\
q^{\varepsilon}=p^{\varepsilon}+\frac{\alpha_{\nu}}{\alpha_{p}} \frac{\partial p^{\varepsilon}}{\partial t}+\chi^{\varepsilon} \gamma_{f}^{\varepsilon}
\end{gathered}
$$

where

$$
\beta^{\varepsilon}=\left\langle\left(1-\chi^{\varepsilon}\right) \operatorname{div}_{x} \boldsymbol{w}^{\varepsilon}\right\rangle_{\Omega}, \quad m \gamma_{f}^{\varepsilon}=\left\langle q^{\varepsilon}\right\rangle_{\Omega}, \quad(1-m) \gamma_{s}^{\varepsilon}=-\frac{1}{\alpha_{\eta}}\left\langle q^{\varepsilon}\right\rangle_{\Omega}+\beta^{\varepsilon} .
$$

The case $\eta_{0}=\infty$ is considered in the same way. Note that for all situations the basic integral identity (1.14) permits to bound only the sum $\left(q^{\varepsilon}+\pi^{\varepsilon}\right)$. But thanks to the property that the product of these two functions is equal to zero, it is enough to get bounds for each of these functions. The pressure $p^{\varepsilon}$ is bounded from the state equation (4.4), if we substitute the term $\left(\alpha_{\nu} / \alpha_{p}\right) \partial p^{\varepsilon} / \partial t$ from the state equation (4.2) and use estimate (1.17).

Estimation of $\boldsymbol{w}^{\varepsilon}$ in the case $\tau_{0}=0$ is not simple, and we outline it in more detail.

Let $\mu_{1}>0$ and $\tau_{0}=0$. As usual, we obtain the basic estimates if we multiply equations for $\boldsymbol{w}^{\varepsilon}$ by $\partial \boldsymbol{w}^{\varepsilon} / \partial t$ and then integrate by parts all obtained terms. The only one term $\boldsymbol{F} \cdot \partial \boldsymbol{w}^{\varepsilon} / \partial t$ heeds additional consideration here. First of all, on the strength of Lemma 3.1, we construct an extension $\boldsymbol{u}^{\varepsilon}$ of the function $\boldsymbol{w}^{\varepsilon}$ from $\Omega_{s}^{\varepsilon}$ into $\Omega_{f}^{\varepsilon}$ such that $\boldsymbol{u}^{\varepsilon}=\boldsymbol{w}^{\varepsilon}$ in $\Omega_{s}^{\varepsilon}, \boldsymbol{u}^{\varepsilon} \in W_{2}^{1}(\Omega)$ and

$$
\left\|\boldsymbol{u}^{\varepsilon}\right\|_{2, \Omega} \leq C\left\|\nabla_{x} \boldsymbol{u}^{\varepsilon}\right\|_{2, \Omega} \leq \frac{C}{\sqrt{\alpha_{\lambda}}}\left\|\left(1-\chi^{\varepsilon}\right) \sqrt{\alpha_{\lambda}} \nabla_{x} \boldsymbol{w}^{\varepsilon}\right\|_{2, \Omega} .
$$

After that we estimate $\left\|\boldsymbol{w}^{\varepsilon}\right\|_{2, \Omega}$ with the help of Friedrichs-Poincaré's inequality in periodic structure (lemma 3.2 ) for the difference $\left(\boldsymbol{u}^{\varepsilon}-\boldsymbol{w}^{\varepsilon}\right)$ :

$$
\begin{gathered}
\left\|\boldsymbol{w}^{\varepsilon}\right\|_{2, \Omega} \leq\left\|\boldsymbol{u}^{\varepsilon}\right\|_{2, \Omega}+\left\|\boldsymbol{u}^{\varepsilon}-\boldsymbol{w}^{\varepsilon}\right\|_{2, \Omega} \leq\left\|\boldsymbol{u}^{\varepsilon}\right\|_{2, \Omega}+C \varepsilon\left\|\chi^{\varepsilon} \nabla_{x}\left(\boldsymbol{u}^{\varepsilon}-\boldsymbol{w}^{\varepsilon}\right)\right\|_{2, \Omega} \\
\leq\left\|\boldsymbol{u}^{\varepsilon}\right\|_{2, \Omega}+C \varepsilon\left\|\nabla_{x} \boldsymbol{u}^{\varepsilon}\right\|_{2, \Omega}+C\left(\varepsilon \alpha_{\mu}^{-\frac{1}{2}}\right)\left\|\chi^{\varepsilon} \sqrt{\alpha_{\mu}} \nabla_{x} \boldsymbol{w}^{\varepsilon}\right\|_{2, \Omega} \\
\leq \frac{C}{\sqrt{\alpha_{\lambda}}}\left\|\left(1-\chi^{\varepsilon}\right) \sqrt{\alpha_{\lambda}} \nabla_{x} \boldsymbol{w}^{\varepsilon}\right\|_{2, \Omega}+C\left(\varepsilon \alpha_{\mu}^{-\frac{1}{2}}\right)\left\|\chi^{\varepsilon} \sqrt{\alpha_{\mu}} \nabla_{x} \boldsymbol{w}^{\varepsilon}\right\|_{2, \Omega} .
\end{gathered}
$$

Next we pass the derivative with respect to time from $\partial \boldsymbol{w}^{\varepsilon} / \partial t$ to $\rho^{\varepsilon} \boldsymbol{F}$ and bound all positive terms (including the term $\alpha_{\nu} \chi^{\varepsilon} \operatorname{div}_{x} \partial \boldsymbol{w}^{\varepsilon} / \partial t$ ) in a usual way with the help of Hölder and Grownwall's inequalities. 
The rest of the proof is the same as for the case $\tau_{0}>0$ if we use a consequence of (1.17):

$$
\max _{0<t<T} \alpha_{\tau}\left\|\frac{\partial^{2} \boldsymbol{w}^{\varepsilon}}{\partial t^{2}}(t)\right\|_{2, \Omega} \leq C
$$

4.2. The proof of this part of the theorem is obvious, because the renormalization reduces this case to the case of $\mu_{1}=1$ and $\tau_{0}=0$, which has been already considered.

4.3. Let $\lambda_{0}=\infty, \mu_{1}<\infty$ and conditions (2.5) hold true. It is obvious that estimates (2.2) are still valid.

The desired estimates (2.6) follow from the basic equations for $\alpha_{\lambda} \boldsymbol{w}^{\varepsilon}$ in the same way as in the case of estimates (2.2). The main difference here is in the term $\rho^{\varepsilon} \boldsymbol{F} \cdot \alpha_{\lambda} \partial \boldsymbol{w}^{\varepsilon} / \partial t$, which now transforms to

$$
\Upsilon \equiv \rho_{f} \boldsymbol{F} \cdot \alpha_{\lambda} \frac{\partial \boldsymbol{w}^{\varepsilon}}{\partial t}+\left(\rho_{f}-\rho_{f}\right)\left(1-\chi^{\varepsilon}\right) \boldsymbol{F} \cdot \alpha_{\lambda} \frac{\partial \boldsymbol{w}^{\varepsilon}}{\partial t} .
$$

The integral of first term in $\Upsilon$ transforms as

$$
\begin{aligned}
& \rho_{f} \int_{0}^{t} \int_{\Omega} \nabla \Phi \cdot \alpha_{\lambda} \frac{\partial \boldsymbol{w}^{\varepsilon}}{\partial \tau} d \boldsymbol{x} d \tau=-\rho_{f} \int_{0}^{t} \int_{\Omega} \Phi \alpha_{\lambda} \operatorname{div}_{x} \frac{\partial \boldsymbol{w}^{\varepsilon}}{\partial \tau} d \boldsymbol{x} d \tau \\
=- & \rho_{f} \int_{0}^{t} \int_{\Omega}\left(\chi^{\varepsilon} \cdot \Phi \alpha_{\lambda} \operatorname{div}_{x} \frac{\partial \boldsymbol{w}^{\varepsilon}}{\partial \tau}+\left(1-\chi^{\varepsilon}\right) \cdot \Phi \alpha_{\lambda} \operatorname{div}_{x} \frac{\partial \boldsymbol{w}^{\varepsilon}}{\partial \tau}\right) d \boldsymbol{x} d \tau \\
= & -\rho_{f} \int_{\Omega}\left(\chi^{\varepsilon} \cdot \Phi \alpha_{\lambda} \operatorname{div}_{x} \boldsymbol{w}^{\varepsilon}+\left(1-\chi^{\varepsilon}\right) \cdot \Phi \alpha_{\lambda} \operatorname{div}_{x} \boldsymbol{u}^{\varepsilon}\right) d \boldsymbol{x}+ \\
& \rho_{f} \int_{0}^{t} \int_{\Omega}\left(\chi^{\varepsilon} \cdot \Phi_{\tau} \alpha_{\lambda} \operatorname{div}_{x} \boldsymbol{w}^{\varepsilon}+\left(1-\chi^{\varepsilon}\right) \cdot \Phi_{\tau} \alpha_{\lambda} \operatorname{div}_{x} \boldsymbol{u}^{\varepsilon}\right) d \boldsymbol{x} d \tau
\end{aligned}
$$

and is bounded with the help of terms

$$
\int_{\Omega}\left(\chi^{\varepsilon}\left(\alpha_{p} \alpha_{\lambda}^{-1}\right)\left(\alpha_{\lambda} \operatorname{div}_{x} \boldsymbol{w}^{\varepsilon}\right)^{2}+\left(1-\chi^{\varepsilon}\right)\left|\alpha_{\lambda} \nabla_{x} \boldsymbol{u}^{\varepsilon}\right|^{2}\right) d \boldsymbol{x},
$$

which appear in the basic identity after using the continuity equations.

The integral of the second term in $\Upsilon$ is bounded with the help of the term

$$
\left\langle\left(1-\chi^{\varepsilon}\right)\left|\alpha_{\lambda} \nabla_{x} \boldsymbol{u}^{\varepsilon}\right|^{2}\right\rangle_{\Omega}
$$

in the same way as before.

Estimates (2.3) follow now from (2.6). Here as before the sum of pressures $\left(q^{\varepsilon}+\pi^{\varepsilon}\right)$ is bounded from the basic integral identity (1.14) as a corresponding functional, and the pressure $p^{\varepsilon}$ is bounded from the equation (4.1) due to the bound (1.17) for the divergence of the velocity of the liquid component $\chi^{\varepsilon} \operatorname{div}_{x}\left(\partial \boldsymbol{w}^{\varepsilon} / \partial t\right)$.

If instead of conditions (2.5) one has condition (2.4), then bounds (2.3) for pressures $p^{\varepsilon}$ and $q^{\varepsilon}$ follow from equations (1.12) and (4.1) and bounds (1.17). Note that in this case $\beta^{\varepsilon}=0$. 


\section{$\S 5$. Proof of Theorem 2.2}

\subsection{Weak and two-scale limits of sequences of displacement} and pressures. On the strength of Theorem 2.1, the sequences $\left\{p^{\varepsilon}\right\},\left\{q^{\varepsilon}\right\}$, $\left\{\pi^{\varepsilon}\right\}$ and $\left\{\boldsymbol{w}^{\varepsilon}\right\}$ are uniformly in $\varepsilon$ bounded in $L^{2}\left(\Omega_{T}\right)$. Hence there exist a subsequence of small parameters $\{\varepsilon>0\}$ and functions $p, q, \pi$ and $\boldsymbol{w}$ such that

$$
p^{\varepsilon} \rightarrow p, \quad q^{\varepsilon} \rightarrow q, \quad \pi^{\varepsilon} \rightarrow \pi, \quad \boldsymbol{w}^{\varepsilon} \rightarrow \boldsymbol{w}
$$

weakly in $L^{2}\left(\Omega_{T}\right)$ as $\varepsilon \searrow 0$.

Due to Lemma 3.1 there is a function $\boldsymbol{u}^{\varepsilon} \in L^{\infty}\left(0, T ; W_{2}^{1}(\Omega)\right)$ such that $\boldsymbol{u}^{\varepsilon}=\boldsymbol{w}^{\varepsilon}$ in $\Omega_{s} \times(0, T)$, and the family $\left\{\boldsymbol{u}^{\varepsilon}\right\}$ is uniformly in $\varepsilon$ bounded in $L^{\infty}\left(0, T ; W_{2}^{1}(\Omega)\right)$. Therefore it is possible to extract a subsequence of $\{\varepsilon>0\}$ such that

$$
\boldsymbol{u}^{\varepsilon} \rightarrow \boldsymbol{u} \text { weakly in } L^{2}\left(0, T ; W_{2}^{1}(\Omega)\right)
$$

as $\varepsilon \searrow 0$. Moreover,

$$
\chi^{\varepsilon} \alpha_{\mu}^{\varepsilon} \mathbb{D}_{x}\left(\boldsymbol{w}^{\varepsilon}\right) \rightarrow 0 .
$$

Relabelling if necessary, we assume that the sequences converge themselves.

On the strength of Nguetseng's theorem, there exist 1-periodic in $\boldsymbol{y}$ functions $P(\boldsymbol{x}, t, \boldsymbol{y}), \Pi(\boldsymbol{x}, t, \boldsymbol{y}), Q(\boldsymbol{x}, t, \boldsymbol{y}), \boldsymbol{W}(\boldsymbol{x}, t, \boldsymbol{y})$ and $\boldsymbol{U}(\boldsymbol{x}, t, \boldsymbol{y})$ such that the sequences $\left\{p^{\varepsilon}\right\},\left\{\pi^{\varepsilon}\right\},\left\{q^{\varepsilon}\right\},\left\{\boldsymbol{w}^{\varepsilon}\right\}$ and $\left\{\nabla_{x} \boldsymbol{u}^{\varepsilon}\right\}$ two-scale converge to $P(\boldsymbol{x}, t, \boldsymbol{y}), \Pi(\boldsymbol{x}, t, \boldsymbol{y}), Q(\boldsymbol{x}, t, \boldsymbol{y}), \boldsymbol{W}(\boldsymbol{x}, t, \boldsymbol{y})$ and $\nabla_{x} \boldsymbol{u}+\nabla_{y} \boldsymbol{U}(\boldsymbol{x}, t, \boldsymbol{y})$, respectively.

Note that the sequence $\left\{\operatorname{div}_{x} \boldsymbol{w}^{\varepsilon}\right\}$ weakly converges to $\operatorname{div}_{x} \boldsymbol{w}$ and $\boldsymbol{u} \in$ $L^{2}\left(0, T ; \stackrel{\circ}{W_{2}^{1}}(\Omega)\right)$. Last assertion for disconnected porous space follows from inclusion $\boldsymbol{u}^{\varepsilon} \in L^{2}\left(0, T ; \stackrel{\circ}{W_{2}^{1}}(\Omega)\right)$ and for the connected porous space it follows from the Friedrichs-Poincaré's inequality for $\boldsymbol{u}^{\varepsilon}$ in the $\varepsilon$-layer of the boundary $S$ and from convergence of sequence $\left\{\boldsymbol{u}^{\varepsilon}\right\}$ to $\boldsymbol{u}$ strongly in $L^{2}\left(\Omega_{T}\right)$ and weakly in $L^{2}\left((0, T) ; W_{2}^{1}(\Omega)\right)$.

\subsection{Micro- and macroscopic equations I.}

Lemma 5.1. For all $\boldsymbol{x} \in \Omega$ and $\boldsymbol{y} \in Y$ weak and two-scale limits of the sequences $\left\{p^{\varepsilon}\right\},\left\{\pi^{\varepsilon}\right\},\left\{q^{\varepsilon}\right\},\left\{\boldsymbol{w}^{\varepsilon}\right\}$, and $\left\{\boldsymbol{u}^{\varepsilon}\right\}$ satisfy the relations

$$
\begin{gathered}
P=\frac{1}{m} \chi p, \quad Q=\frac{1}{m} \chi q ; \\
\frac{1}{\eta_{0}} \Pi+(1-\chi)\left(d i v_{x} \boldsymbol{u}+d i v_{y} \boldsymbol{U}\right)=\gamma_{s}(1-\chi) ; \\
\operatorname{div}_{y} \boldsymbol{W}=0 ; \\
\boldsymbol{W}=\chi(\boldsymbol{y}) \boldsymbol{W}+(1-\chi) \boldsymbol{u} ; \\
q=p+\nu_{0} p_{*}^{-1} \frac{\partial p}{\partial t}+\gamma_{f} m ; \\
\frac{1}{p_{*}} p+d i v_{x} \boldsymbol{w}=(1-m) d i v_{x} \boldsymbol{u}+\left\langle\operatorname{div}_{y} \boldsymbol{U}\right\rangle_{Y_{s}}-\beta ; \\
\frac{1}{\eta_{0}} \pi+(1-m) d i v_{x} \boldsymbol{u}+\left\langle d i v_{y} \boldsymbol{U}\right\rangle_{Y_{s}}=(1-m) \gamma_{s},
\end{gathered}
$$


where

$$
\beta=\int_{\Omega}\left\langle\operatorname{div}_{y} \boldsymbol{U}\right\rangle_{Y_{s}} d x, \quad m \gamma_{f}=\langle q\rangle_{\Omega}, \quad(1-m) \gamma_{s}=-\frac{1}{\eta_{0}}\langle q\rangle_{\Omega}+\beta,
$$

if $p_{*}+\eta_{0}=\infty$ and $\beta=\gamma_{f}=\gamma_{s}=0$, if $p_{*}+\eta_{0}<\infty$.

Proof. In order to prove Eq. (5.4), into Eq. (1.14) insert a test function $\boldsymbol{\psi}^{\varepsilon}=\varepsilon \boldsymbol{\psi}(\boldsymbol{x}, t, \boldsymbol{x} / \varepsilon)$, where $\boldsymbol{\psi}(\boldsymbol{x}, t, \boldsymbol{y})$ is an arbitrary 1-periodic and finite on $Y_{f}$ function in $\boldsymbol{y}$. Passing to the limit as $\varepsilon \searrow 0$, we get

$$
\nabla_{y} Q(\boldsymbol{x}, t, \boldsymbol{y})=0, \quad \boldsymbol{y} \in Y_{f} .
$$

The weak and two-scale limiting passage in Eq. (4.4) yield that Eq. (5.8) and the equation

$$
Q=P+\frac{\nu_{0}}{p_{*}} \frac{\partial P}{\partial t}+\gamma_{f} \chi
$$

hold. Taking into account Eq. (5.11) and (5.12) we get

$$
\nabla_{y} P(\boldsymbol{x}, t, \boldsymbol{y})=0, \quad \boldsymbol{y} \in Y_{f} .
$$

Next, fulfilling the two-scale limiting passage in equalities

$$
\left(1-\chi^{\varepsilon}\right) p^{\varepsilon}=0, \quad\left(1-\chi^{\varepsilon}\right) q^{\varepsilon}=0
$$

we arrive at

$$
(1-\chi) P=0, \quad(1-\chi) Q=0,
$$

which along with Eqs. (5.11) and (5.12) justifies Eq. (5.4).

Eqs. (5.5), (5.6), (5.9), and (5.10) appear as the results of two-scale limiting passages in Eqs. (4.2) - (4.4) with the proper test functions being involved. Thus, for example, Eq. (5.9) arises if we represent Eq. (4.2) in the form

$$
p^{\varepsilon}+\operatorname{div}_{x} \boldsymbol{w}^{\varepsilon}=\left(1-\chi^{\varepsilon}\right) \operatorname{div}_{x} \boldsymbol{u}^{\varepsilon}-\frac{1}{m} \beta^{\varepsilon} \chi^{\varepsilon},
$$

multiply by an arbitrary function, independent of the "fast" variable $\boldsymbol{x} / \varepsilon$, and then pass to the limit as $\varepsilon \searrow 0$. Eq. (5.10) is derived quite similarly. In order to prove Eq. (5.6), it is sufficient to consider the two-scale limiting relations in Eq. (5.13) as $\varepsilon \searrow 0$ with the test functions $\varepsilon \psi(\boldsymbol{x} / \varepsilon) h(\boldsymbol{x}, t)$, where $\psi$ and $h$ are arbitrary smooth test functions. In order to prove Eq. (5.7) it is sufficient to consider the two-scale limiting relations in

$$
\left(1-\chi^{\varepsilon}\right)\left(\boldsymbol{w}^{\varepsilon}-\boldsymbol{u}^{\varepsilon}\right)=0 .
$$


Lemma 5.2. For all $(\boldsymbol{x}, t) \in \Omega_{T}$ the relations

$$
\begin{aligned}
& \lambda_{0} \triangle_{y} \boldsymbol{U}=\nabla_{y} \Pi, \quad \boldsymbol{y} \in Y_{s}, \\
& \left(\lambda_{0} \mathbb{D}(y, \boldsymbol{U})-\Pi \cdot \mathbb{I}+\lambda_{0} \mathbb{D}(x, \boldsymbol{u})+\frac{1}{m} q \cdot \mathbb{I}\right) \cdot \boldsymbol{n}=0, \quad \boldsymbol{y} \in \gamma,
\end{aligned}
$$

hold true. Here $\boldsymbol{n}$ is a unit normal to $\gamma$.

Proof. Substituting a test function of the form $\boldsymbol{\psi}^{\varepsilon}=\varepsilon \boldsymbol{\psi}(\boldsymbol{x}, t, \boldsymbol{x} / \varepsilon)$, where $\boldsymbol{\psi}(\boldsymbol{x}, t, \boldsymbol{y})$ is an arbitrary 1-periodic in $\boldsymbol{y}$ function vanishing on the boundary $S$, into Eq.(1.14), and passing to the limit as $\varepsilon \searrow 0$, we arrive at the following microscopic relation on the cell $Y$ :

$$
\operatorname{div}_{y}\left\{\lambda_{0}(1-\chi)(\mathbb{D}(y, \boldsymbol{U})+\mathbb{D}(x, \boldsymbol{u}))-\left(\Pi+\frac{1}{m} q \chi\right) \cdot \mathbb{I}\right\}=0,
$$

which is clearly equivalent to Eqs. (5.14) and (5.15) in view of Eqs.(5.4).

Lemma 5.3. Let $\hat{\rho}=m \rho_{f}+(1-m) \rho_{s}, \boldsymbol{V}=\chi \partial \boldsymbol{w} / \partial t$, and $\boldsymbol{v}=\langle\boldsymbol{V}\rangle_{Y}$. Then for all $0 \leq \tau_{0}<\infty$ the quadruple of functions $\{\boldsymbol{u}, \boldsymbol{v}, q, \pi\}$ satisfies in $\Omega_{T}$ the system of macroscopic equations

$$
\begin{aligned}
& \tau_{0} \rho_{f} \frac{\partial \boldsymbol{v}}{\partial t}+\tau_{0} \rho_{s}(1-m) \frac{\partial^{2} \boldsymbol{u}}{\partial t^{2}}-\hat{\rho} \boldsymbol{F}= \\
& \operatorname{div}_{x}\left\{\lambda_{0}\left((1-m) \mathbb{D}(x, \boldsymbol{u})+\langle\mathbb{D}(y, \boldsymbol{U})\rangle_{Y_{s}}\right)-(q+\pi) \cdot \mathbb{I}\right\} .
\end{aligned}
$$

Proof. Eqs. (5.17) arise as the limit of Eqs. (1.14) with test functions being finite in $\Omega_{T}$ and independent of $\varepsilon$.

\subsection{Micro- and macroscopic equations II.}

Lemma 5.4. If $\mu_{1}=\infty$ then the weak and two-scale limits of $\left\{\boldsymbol{u}^{\varepsilon}\right\}$ and $\left\{\boldsymbol{w}^{\varepsilon}\right\}$ coincide.

Proof. In order to verify, it is sufficient to consider the difference $\left(\boldsymbol{u}^{\varepsilon}-\boldsymbol{w}^{\varepsilon}\right)$ and apply Friedrichs-Poincar'e's inequality, just like in the proof of Theorem 2.1 .

Lemma 5.5. Let $\mu_{1}<\infty$. Then the weak and two-scale limits of $\left\{q^{\varepsilon}\right\}$ and $\left\{\chi^{\varepsilon} \boldsymbol{w}^{\varepsilon}\right\}$ satisfy the microscopic relations

$$
\begin{gathered}
\tau_{0} \rho_{f} \frac{\partial \boldsymbol{V}}{\partial t}-\rho_{f} \boldsymbol{F}=\mu_{1} \triangle_{y} \boldsymbol{V}-\nabla_{y} R-\nabla_{x} q, \quad \boldsymbol{y} \in Y_{f} \\
\boldsymbol{V}=\frac{\partial \boldsymbol{u}}{\partial t}, \quad \boldsymbol{y} \in \gamma
\end{gathered}
$$

in the case $\mu_{1}>0$, and relations

$$
\tau_{0} \rho_{f} \frac{\partial \boldsymbol{V}}{\partial t}=-\nabla_{y} R-\nabla_{x} q+\rho_{f} \boldsymbol{F}, \quad \boldsymbol{y} \in Y_{f}
$$




$$
\left(\boldsymbol{V}-\frac{\partial \boldsymbol{u}}{\partial t}\right) \cdot \boldsymbol{n}=0, \quad \boldsymbol{y} \in \gamma
$$

in the case $\mu_{1}=0$.

In Eq. (5.21) $\boldsymbol{n}$ is the unit normal to $\gamma$.

Proof. Differential equations (5.18) and (5.20) follow as $\varepsilon \searrow 0$ from integral equality (1.14) with the test function $\boldsymbol{\psi}=\boldsymbol{\varphi}\left(x \varepsilon^{-1}\right) \cdot h(\boldsymbol{x}, t)$, where $\boldsymbol{\varphi}$ is solenoidal and finite in $Y_{f}$.

Boundary conditions (5.19) are the consequences of the two-scale convergence of $\left\{\alpha_{\mu}^{\frac{1}{2}} \nabla_{x} \boldsymbol{w}^{\varepsilon}\right\}$ to the function $\mu_{1}^{\frac{1}{2}} \nabla_{y} \boldsymbol{W}(\boldsymbol{x}, t, \boldsymbol{y})$. On the strength of this convergence, the function $\nabla_{y} \boldsymbol{W}(\boldsymbol{x}, t, \boldsymbol{y})$ is $L^{2}$-integrable in $Y$. The boundary conditions (5.21) follow from Eq. (5.6).

Lemma 5.6. If the porous space is disconnected, which is the case of isolated pores, then the weak and two-scale limits of sequences $\left\{\boldsymbol{u}^{\varepsilon}\right\}$ and $\left\{\boldsymbol{w}^{\varepsilon}\right\}$ coincide.

Proof. Indeed, in the case $0 \leq \mu_{1}<\infty$ the systems of equations (5.6), (5.18), and (5.19), or (5.6), (5.20), and (5.21) have the unique solution $\boldsymbol{V}=$ $\partial \boldsymbol{u} / \partial t$

\subsection{Homogenized equations I.}

Lemma 5.7. If $\mu_{1}=\infty$ or the porous space is disconnected then $\boldsymbol{w}=\boldsymbol{u}$ and the weak limits $\boldsymbol{u}, p, q$, and $\pi$ satisfy in $\Omega_{T}$ the initial-boundary value problem

$$
\begin{gathered}
\tau_{0} \hat{\rho} \frac{\partial^{2} \boldsymbol{u}}{\partial t^{2}}=\operatorname{div}_{x}\left\{\lambda_{0} \mathbb{A}_{0}^{s}: \mathbb{D}(x, \boldsymbol{u})+B_{0}^{s} d i v_{x} \boldsymbol{u}+B_{1}^{s} q-(q+\pi) \cdot \mathbb{I}\right\}+\hat{\rho} \boldsymbol{F} \\
\frac{1}{\eta_{0}} \pi+C_{0}^{s}: \mathbb{D}(x, \boldsymbol{u})+a_{0}^{s} d i v_{x} \boldsymbol{u}+a_{1}^{s} q+a_{2}^{s}\langle q\rangle_{\Omega}=0 \\
q=p+\nu_{0} p_{*}^{-1} \frac{\partial p}{\partial t}+\gamma_{f} m, \quad \frac{1}{p_{*}} p+\frac{1}{\eta_{0}} \pi+\operatorname{div}_{x} \boldsymbol{u}=(1-m) \gamma_{s}-\beta
\end{gathered}
$$

where the symmetric strictly positively defined constant fourth-rank tensor $\mathbb{A}_{0}^{s}$, matrices $C_{0}^{s}, B_{0}^{s}$ and $B_{1}^{s}$ and constants $a_{0}^{s}, a_{1}^{s}$ and $a_{2}^{s}$ are defined below by formulas (5.30), (5.32) - (5.34).

Differential equations (5.22) are endowed with homogeneous initial and boundary conditions

$$
\tau_{0} \boldsymbol{u}(\boldsymbol{x}, 0)=\tau_{0} \frac{\partial \boldsymbol{u}}{\partial t}(\boldsymbol{x}, 0)=0, \quad \boldsymbol{x} \in \Omega, \quad \boldsymbol{u}(\boldsymbol{x}, t)=0, \quad \boldsymbol{x} \in S, \quad t>0 .
$$

Remark 5.1. In what follows we can neglect terms $a_{2}^{s}\langle q\rangle_{\Omega}, \gamma_{f}$ and $\gamma_{s}$ because all pressures are defined up to arbitrary functions of time. 
Proof. In the first place let us notice that $\boldsymbol{u}=\boldsymbol{w}$ due to Lemmas 5.4 and 5.6 .

The homogenized equations (5.22) follow from the macroscopic equations (5.17), after we insert in them the expression

$$
\langle\mathbb{D}(y, \boldsymbol{U})\rangle_{Y_{s}}=\mathbb{A}_{1}^{s}: \mathbb{D}(x, \boldsymbol{u})+B_{0}^{s} \operatorname{div}_{x} \boldsymbol{u}+B_{1}^{s} q .
$$

In turn, this expression follows by virtue of solutions of Eqs. (5.5) and (5.16) on the pattern cell $Y_{s}$. Indeed, setting

$$
\begin{aligned}
& \boldsymbol{U}=\sum_{i, j=1}^{3} \boldsymbol{U}^{i j}(\boldsymbol{y}) D_{i j}+\boldsymbol{U}_{0}(\boldsymbol{y}) \operatorname{div}_{x} \boldsymbol{u}+\frac{1}{m}\left(\boldsymbol{U}_{1}(\boldsymbol{y})\left(q-\langle q\rangle_{\Omega}\right)+\boldsymbol{U}_{2}(\boldsymbol{y})\langle q\rangle_{\Omega}\right), \\
& \Pi=\lambda_{0} \sum_{i, j=1}^{3} \Pi^{i j}(\boldsymbol{y}) D_{i j}+\Pi_{0}(\boldsymbol{y}) \operatorname{div}_{x} \boldsymbol{u}+\frac{1}{m}\left(\Pi_{1}(\boldsymbol{y})\left(q-\langle q\rangle_{\Omega}\right)+\Pi_{2}(\boldsymbol{y})\langle q\rangle_{\Omega}\right),
\end{aligned}
$$

where

$$
D_{i j}(\boldsymbol{x}, t)=\frac{1}{2}\left(\frac{\partial u_{i}}{\partial x_{j}}(\boldsymbol{x}, t)+\frac{\partial u_{j}}{\partial x_{i}}(\boldsymbol{x}, t)\right),
$$

we arrive at the following periodic-boundary value problems in $Y_{s}$ :

$$
\left.\begin{array}{r}
\operatorname{div}_{y}\left\{(1-\chi)\left(\mathbb{D}\left(y, \boldsymbol{U}^{i j}\right)+J^{i j}\right)-\Pi^{i j} \cdot \mathbb{I}\right\}=0, \\
\frac{\lambda_{0}}{\eta_{0}} \Pi^{i j}+(1-\chi) \operatorname{div}_{y} \boldsymbol{U}^{i j}=0 ; \\
\operatorname{div}_{y}\left\{\lambda_{0}(1-\chi) \mathbb{D}\left(y, \boldsymbol{U}_{0}\right)-\Pi_{0} \cdot \mathbb{I}\right\}=0, \\
\frac{1}{\eta_{0}} \Pi_{0}+(1-\chi)\left(\operatorname{div}_{y} \boldsymbol{U}_{0}+1\right)=0 ;
\end{array}\right\}
$$

Note that for $p_{*}+\eta_{0}=\infty$

$$
\begin{aligned}
& \beta=\left\langle\left\langle\operatorname{div}_{y} \boldsymbol{U}\right\rangle_{\Omega}\right\rangle_{Y_{s}}=\sum_{i, j=1}^{3}\left\langle\operatorname{div}_{y} \boldsymbol{U}^{i j}\right\rangle_{Y_{s}}\left\langle D_{i j}\right\rangle_{\Omega}+\left\langle\operatorname{div}_{y} \boldsymbol{U}_{0}\right\rangle_{Y_{s}}\left\langle\operatorname{div}_{x} \boldsymbol{u}\right\rangle_{\Omega}+ \\
& \frac{1}{m}\left\langle\operatorname{div}_{y} \boldsymbol{U}_{1}\right\rangle_{Y_{s}}\left\langle\left(q-\langle q\rangle_{\Omega}\right)\right\rangle_{\Omega}+\frac{1}{m}\left\langle\operatorname{div}_{y} \boldsymbol{U}_{2}\right\rangle_{Y_{s}}\langle q\rangle_{\Omega}=\frac{1}{m}\left\langle\operatorname{div}_{y} \boldsymbol{U}_{2}\right\rangle_{Y_{s}}\langle q\rangle_{\Omega}
\end{aligned}
$$

due to homogeneous boundary conditions for $\boldsymbol{u}(\boldsymbol{x}, t)$.

On the strength of the assumptions on the geometry of the pattern "liquid" cell $Y_{s}$, problems (5.26) - (??) have unique solution, up to an arbitrary 
constant vector. In order to discard the arbitrary constant vectors we demand

$$
\left\langle\boldsymbol{U}^{i j}\right\rangle_{Y_{s}}=\left\langle\boldsymbol{U}_{0}\right\rangle_{Y_{s}}=\left\langle\boldsymbol{U}_{1}\right\rangle_{Y_{s}}=\left\langle\boldsymbol{U}_{2}\right\rangle_{Y_{s}}=0
$$

Thus

$$
\mathbb{A}_{0}^{s}=\sum_{i, j=1}^{3} J^{i j} \otimes J^{i j}+\mathbb{A}_{1}^{s}, \quad \mathbb{A}_{1}^{s}=\sum_{i, j=1}^{3}\left\langle(1-\chi) D\left(y, U^{i j}\right)\right\rangle_{Y} \otimes J^{i j}
$$

Symmetry of the tensor $\mathbb{A}_{0}^{s}$ follows from symmetry of the tensor $\mathbb{A}_{1}^{s}$. And symmetry of the latter one follows from the equality

$$
\begin{aligned}
& \left\langle\mathbb{D}\left(y, \boldsymbol{U}^{i j}\right)\right\rangle_{Y_{s}}: J^{k l}= \\
& -\left\langle\mathbb{D}\left(y, \boldsymbol{U}^{i j}\right): \mathbb{D}\left(y, \boldsymbol{U}^{k l}\right)\right\rangle_{Y_{s}}+\frac{\lambda_{0}}{\eta_{0}} \Pi^{i j} \Pi^{k l},
\end{aligned}
$$

which appears by means of multiplication of Eq. (5.26) for $\boldsymbol{U}^{i j}$ by $\boldsymbol{U}^{k l}$ and by integration by parts.

This equality also implies positive definiteness of the tensor $\mathbb{A}_{0}^{s}$. Indeed, let $\zeta$ be an arbitrary symmetric matrix. Setting

$$
\mathbb{Z}=\sum_{i, j=1}^{3} \boldsymbol{U}^{i j} \zeta_{i j}, \quad \tilde{\Pi}=\sum_{i, j=1}^{3} \Pi^{i j} \zeta_{i j}
$$

and taking into account Eq. (5.31) we get

$$
\langle\mathbb{D}(y, \mathbb{Z})\rangle_{Y_{s}}: \zeta=-\langle\mathbb{D}(y, \mathbb{Z}): \mathbb{D}(y, \mathbb{Z})\rangle_{Y_{s}}-\frac{\lambda_{0}}{\eta_{0}} \tilde{\Pi}^{2}
$$

This equality and the definition of the tensor $A_{0}^{s}$ give

$$
\left(A_{0}^{s}: \zeta\right): \zeta=\langle(\mathbb{D}(y, \mathbb{Z})+\zeta):(\mathbb{D}(y, \mathbb{Z})+\zeta)\rangle_{Y_{s}}+\frac{\lambda_{0}}{\eta_{0}} \tilde{\Pi}^{2}
$$

Now the strict positive definiteness of the tensor $\mathbb{A}_{0}$ follows from the equality immediately above and the geometry of the elementary cell $Y_{s}$. Namely, let for some function $\zeta$ such that $\zeta: \zeta=1$ be $\left(\mathbb{A}_{0}: \zeta\right): \zeta=0$. But then we have $(\mathbb{D}(y, \mathbb{Z})+\zeta)=0$, which is possible iff $\mathbb{Z}$ is a linear function in $\boldsymbol{y}$. On the other hand, all linear periodic functions on $Y_{s}$ are constant. Finally, the normalization condition $\left\langle\boldsymbol{U}^{i j}\right\rangle=0$ yields that $\mathbb{Z}=0$. However, this is impossible because the functions $\boldsymbol{U}^{i j}$ are linearly independent.

Finally, Eqs. (5.23) and (5.24) for the pressures follow from Eqs. (5.8)(5.10) and

$$
B_{0}^{s}=\left\langle\mathbb{D}\left(y, \boldsymbol{U}_{0}\right)\right\rangle_{Y_{s}}, \quad B_{1}^{s}=\frac{1}{m}\left\langle\mathbb{D}\left(y, \boldsymbol{U}_{1}\right)\right\rangle_{Y_{s}}
$$




$$
\begin{aligned}
C_{0}^{s}= & \sum_{i, j=1}^{3}\left\langle\operatorname{div}_{y} \boldsymbol{U}^{i j}\right\rangle_{Y_{s}} J^{i j}, \quad a_{0}^{s}=1-m+\left\langle\operatorname{div}_{y} \boldsymbol{U}_{0}\right\rangle_{Y_{s}}, \\
& a_{1}^{s}=\frac{1}{m}\left\langle\operatorname{div}_{y} \boldsymbol{U}_{1}\right\rangle_{Y_{s}}, \quad a_{2}^{s}=\frac{1}{m}\left(\frac{m}{\eta_{0}}-\left\langle\operatorname{div}_{y} \boldsymbol{U}_{1}\right\rangle_{Y_{s}}\right) .
\end{aligned}
$$

\subsection{Homogenized equations II.}

Let $\mu_{1}<\infty$. In the same manner as above, we verify that the limit $\boldsymbol{u}$ of the sequence $\left\{\boldsymbol{u}^{\varepsilon}\right\}$ satisfies the initial-boundary value problem likes (5.22)(5.24). The main difference here that, in general, the weak limit $\boldsymbol{w}$ of the sequence $\left\{\boldsymbol{W}^{\varepsilon}\right\}$ differs from $\boldsymbol{u}$. More precisely, the following statement is true.

Lemma 5.8. If $\mu_{1}<\infty$ then the weak limits $\boldsymbol{u}, \boldsymbol{w}^{f}, p, q$, and $\pi$ of the sequences $\left\{\boldsymbol{u}^{\varepsilon}\right\},\left\{\chi^{\varepsilon} \boldsymbol{W}^{\varepsilon}\right\},\left\{p^{\varepsilon}\right\},\left\{q^{\varepsilon}\right\}$, and $\left\{\pi^{\varepsilon}\right\}$ satisfy the initial-boundary value problem in $\Omega_{T}$, consisting of the balance of momentum equation

$$
\begin{aligned}
& \tau_{0}\left(\rho_{f} \frac{\partial \boldsymbol{v}}{\partial t}+\rho_{s}(1-m) \frac{\partial^{2} \boldsymbol{u}}{\partial t^{2}}\right)+\nabla(q+\pi)-\hat{\rho} \boldsymbol{F}= \\
& \operatorname{div}_{x}\left\{\lambda_{0} A_{0}^{s}: \mathbb{D}(x, \boldsymbol{u})+B_{0}^{s} d i v_{x} \boldsymbol{u}+B_{1}^{s} q\right\},
\end{aligned}
$$

where $\boldsymbol{v}=\partial \boldsymbol{w}^{f} / \partial t$ and $\mathbb{A}_{0}^{s}, B_{0}^{s}$ and $B_{1}^{s}$ are the same as in (2.7), the continuity equations (5.12), the equations

$$
p+\nu_{0} p_{*}^{-1} \frac{\partial p}{\partial t}=q, \quad \frac{1}{p_{*}} p+\frac{1}{\eta_{0}} \pi+\operatorname{div}_{x} \boldsymbol{w}^{f}=(m-1) d i v_{x} \boldsymbol{u},
$$

and Darcy's law in the form

$$
\boldsymbol{v}=m \frac{\partial \boldsymbol{u}}{\partial t}+\int_{0}^{t} B_{1}\left(\mu_{1}, t-\tau\right) \cdot\left(-\nabla_{x} q+\rho_{f} \boldsymbol{F}-\tau_{0} \rho_{f} \frac{\partial^{2} \boldsymbol{u}}{\partial \tau^{2}}\right)(\boldsymbol{x}, \tau) d \tau
$$

in the case of $\tau_{0}>0$ and $\mu_{1}>0$, Darcy's law in the form

$$
\boldsymbol{v}=m \frac{\partial \boldsymbol{u}}{\partial t}+B_{2}\left(\mu_{1}\right) \cdot\left(-\nabla_{x} q+\rho_{f} \boldsymbol{F}\right)
$$

in the case of $\tau_{0}=0$ and $\mu_{1}>0$, and, finally, Darcy's law in the form

$$
\boldsymbol{v}=B_{3} \cdot \frac{\partial \boldsymbol{u}}{\partial t}+\frac{1}{\tau_{0} \rho_{f}}\left(m \mathbb{I}-B_{3}\right) \cdot \int_{0}^{t}\left(-\nabla_{x} q(\boldsymbol{x}, \tau)+\rho_{f} \boldsymbol{F}(\boldsymbol{x}, \tau)\right) d \tau
$$

in the case of $\tau_{0}>0$ and $\mu_{1}=0$. The problem is supplemented by boundary and initial conditions (5.24) for the displacement $\boldsymbol{u}$ of the rigid component and by the boundary condition

$$
\boldsymbol{v}(\boldsymbol{x}, t) \cdot \boldsymbol{n}(\boldsymbol{x})=0, \quad(\boldsymbol{x}, t) \in S=\partial \Omega, \quad t>0,
$$

for the velocity $\boldsymbol{v}$ of the liquid component. In Eqs. (5.37)-(5.40) $\boldsymbol{n}(\boldsymbol{x})$ is the unit normal vector to $S$ at a point $\boldsymbol{x} \in S$, and matrices $B_{1}\left(\mu_{1}, t\right), B_{2}\left(\mu_{1}\right)$, and $B_{3}$ are given below by Eqs. (5.42) -(5.46) . 
Proof. The derivation of Eq. (5.40) is standard [3]. The homogenized equations of balance of momentum and balance of mass derive exactly as (5.22)-(5.23). For example, to get Eq. (5.36) we just expressed $\operatorname{div}_{x} \boldsymbol{w}$ in a sum of Eqs. (5.9) and (5.10) using Eq. (5.7) after homogenization: $\boldsymbol{w}=\boldsymbol{w}^{f}+(1-m) \boldsymbol{u}$. Therefore we omit the relevant proofs now and focus only on derivation of homogenized equations for the velocity $\boldsymbol{v}$ in the form of Darcy's laws.

a) If $\mu_{1}>0$ and $\tau_{0}>0$, then the solution of the system of microscopic equations (5.6), (5.18), and (5.19), provided with the homogeneous initial data, is given by formula

$$
\boldsymbol{V}=\frac{\partial \boldsymbol{u}}{\partial t}+\int_{0}^{t} \mathbf{B}_{1}^{f}(\boldsymbol{y}, t-\tau) \cdot\left(-\nabla_{x} q+\rho_{f} \boldsymbol{F}-\tau_{0} \rho_{f} \frac{\partial^{2} \boldsymbol{u}}{\partial \tau^{2}}\right)(\boldsymbol{x}, \tau) d \tau,
$$

in which

$$
\mathbf{B}_{1}^{f}(\boldsymbol{y}, t)=\sum_{i=1}^{3} \boldsymbol{V}^{i}(\boldsymbol{y}, t) \otimes \boldsymbol{e}_{i}
$$

and the functions $\boldsymbol{V}^{i}(\boldsymbol{y}, t)$ are defined by virtue of the periodic initial-boundary value problem

$$
\left.\begin{array}{l}
\tau_{0} \rho_{f} \frac{\partial \boldsymbol{V}^{i}}{\partial t}-\mu_{1} \triangle \boldsymbol{V}^{i}+\nabla Q^{i}=0, \quad \operatorname{div}_{y} \boldsymbol{V}^{i}=0, \quad \boldsymbol{y} \in Y_{f}, t>0 \\
\boldsymbol{V}^{i}=0, \quad \boldsymbol{y} \in \gamma, t>0 ; \quad \tau_{0} \rho_{f} \boldsymbol{V}^{i}(y, 0)=\boldsymbol{e}_{i}, \quad \boldsymbol{y} \in Y_{f}
\end{array}\right\}
$$

In Eq. (5.41) $\boldsymbol{e}_{i}$ is the standard Cartesian basis vector.

Therefore

$$
B_{1}\left(\mu_{1}, t\right)=\left\langle\mathbf{B}_{1}^{f}(\boldsymbol{y}, t)\right\rangle_{Y_{s}},
$$

b) If $\tau_{0}=0$ and $\mu_{1}>0$ then the solution of the stationary microscopic equations (5.6), (5.18), and (5.19) is given by formula

$$
\boldsymbol{V}=\frac{\partial \boldsymbol{u}}{\partial t}+\mathbf{B}_{2}^{f}(\boldsymbol{y}) \cdot\left(-\nabla q+\rho_{f} \boldsymbol{F}\right)
$$

in which

$$
\mathbf{B}_{2}^{f}(\boldsymbol{y})=\sum_{i=1}^{3} \boldsymbol{U}^{i}(\boldsymbol{y}) \otimes \boldsymbol{e}_{i}
$$

and the functions $\boldsymbol{U}^{i}(\boldsymbol{y})$ are defined from the periodic boundary value problem

$$
\left.\begin{array}{l}
-\mu_{1} \triangle \boldsymbol{U}^{i}+\nabla R^{i}=\boldsymbol{e}_{i}, \quad \operatorname{div}_{y} \boldsymbol{U}^{i}=0, \quad \boldsymbol{y} \in Y_{f}, \\
\boldsymbol{U}^{i}=0, \quad \boldsymbol{y} \in \gamma
\end{array}\right\}
$$

Thus

$$
B_{2}\left(\mu_{1}\right)=\left\langle\mathbf{B}_{2}^{f}((\boldsymbol{y})\rangle_{Y_{s}}\right.
$$


Matrices $B_{1}\left(\mu_{1}, t\right)$ and $B_{2}\left(\mu_{1}\right)$ are symmetric and positively defined [3. Chap. 8].

c) If $\tau_{0}>0$ and $\mu_{1}=0$ then in the process of solving the system (5.6), (5.20), and (5.21) we firstly find the pressure $R(\boldsymbol{x}, t, \boldsymbol{y})$ by virtue of solving the Neumann problem for Laplace's equation in $Y_{f}$. If

$$
\boldsymbol{h}(\boldsymbol{x}, t)=-\tau_{0} \rho_{f} \frac{\partial^{2} \boldsymbol{u}}{\partial t^{2}}(\boldsymbol{x}, t)-\nabla_{x} q(\boldsymbol{x}, t)+\rho_{f} \boldsymbol{F}(\boldsymbol{x}, t)
$$

then

$$
R(\boldsymbol{x}, t, \boldsymbol{y})=\sum_{i=1}^{3} R_{i}(\boldsymbol{y}) \boldsymbol{e}_{i} \otimes \boldsymbol{h}(\boldsymbol{x}, t),
$$

where $R^{i}(\boldsymbol{y})$ is the solution of the problem

$$
\triangle_{y} R_{i}=0, \quad \boldsymbol{y} \in Y_{f} ; \quad \nabla_{y} R_{i} \cdot \boldsymbol{n}=\boldsymbol{n} \cdot \boldsymbol{e}_{i}, \quad \boldsymbol{y} \in \gamma
$$

Formula (5.39) appears as the result of homogenization of Eqs. (5.20) and

$$
B_{3}=\sum_{i=1}^{3}\left\langle\nabla R_{i}(\boldsymbol{y})\right\rangle_{Y_{s}} \otimes \boldsymbol{e}_{i}
$$

where the matrix $\left(m \mathbb{I}-B_{3}\right)$ is symmetric and positively definite [3, Chap. $8]$.

\section{§6. Proof of Theorem 2.3}

\subsection{Weak and two-scale limits of sequences of displacement and pressures.}

I. Let $\mu_{1}<\infty$ and one of the conditions (2.4) or (2.5) holds true. Then on the strength of Theorems 2.1 and 3.1 we conclude that sequences $\left\{\chi^{\varepsilon} \boldsymbol{w}^{\varepsilon}\right\}$, $\left\{p^{\varepsilon}\right\}$ and $\left\{q^{\varepsilon}\right\}$ two-scale converge to $\chi(\boldsymbol{y}) \boldsymbol{W}(\boldsymbol{x}, t, \boldsymbol{y}), P(\boldsymbol{x}, t, \boldsymbol{y})$ and $Q(\boldsymbol{x}, t, \boldsymbol{y})$ and weakly converge in $L^{2}\left(\Omega_{T}\right)$ to $\boldsymbol{w}^{f}, p$ and $q$ respectively, and a sequence $\left\{\boldsymbol{u}^{\varepsilon}(\boldsymbol{x}, t)\right\}$, where $\boldsymbol{u}^{\varepsilon}(\boldsymbol{x}, t)$ is an extension of $\boldsymbol{w}^{\varepsilon}(\boldsymbol{x}, t)$ from the domain $\Omega_{s}^{\varepsilon}$ into domain $\Omega$, strongly converges in $L^{2}\left(\Omega_{T}\right)$ and weakly in $L^{2}\left((0, T) ; W_{2}^{1}(\Omega)\right)$ to zero.

II. If $\mu_{1}<\infty$ and conditions (2.5) hold true, then due to estimates (2.3) and (2.6) the sequence $\left\{\alpha_{\lambda} \boldsymbol{u}^{\varepsilon}\right\}$ converges strongly in $L^{2}\left(\Omega_{T}\right)$ and weakly in $L^{2}\left((0, T) ; W_{2}^{1}(\Omega)\right)$ to a function $\boldsymbol{u}$, and the sequence $\left\{\pi^{\varepsilon}\right\}$ converges weakly in $L^{2}\left(\Omega_{T}\right)$ to a function $\pi$.

III. If $\mu_{1}=\infty, p_{1}^{-1}, \eta_{1}^{-1}<\infty$ and $0<\lambda_{1}<\infty$, then on the strength of part 2) of Theorem 2.1 the sequences $\left\{\alpha_{\mu} \varepsilon^{-2} \chi^{\varepsilon} \boldsymbol{w}^{\varepsilon}\right\},\left\{p^{\varepsilon}\right\},\left\{\pi^{\varepsilon}\right\}$ and $\left\{q^{\varepsilon}\right\}$ two-scale converge to functions $\chi(\boldsymbol{y}) \boldsymbol{W}(\boldsymbol{x}, t, \boldsymbol{y}), P(\boldsymbol{x}, t, \boldsymbol{y}), \Pi(\boldsymbol{x}, t, \boldsymbol{y})$ and $Q(\boldsymbol{x}, t, \boldsymbol{y})$ and weakly in $L^{2}\left(\Omega_{T}\right)$ to functions $\boldsymbol{w}^{f}, p, \pi$ and $q$ respectively, 
and the sequence $\left\{\alpha_{\mu} \varepsilon^{-2} \boldsymbol{u}^{\varepsilon}\right\}$ strongly converge in $L^{2}\left(\Omega_{T}\right)$ and weakly in $L^{2}\left((0, T) ; W_{2}^{1}(\Omega)\right)$ to the function $\boldsymbol{u}$.

As before in previous section $\S 5$, we conclude that $\boldsymbol{u} \in L^{2}\left(0, T ; \stackrel{\circ}{W_{2}^{1}}(\Omega)\right)$.

\subsection{Homogenized equations.}

I. If $\mu_{1}<\infty$ and one of the conditions (2.4) or (2.5) holds true, then, as in the proof of Theorem 2.2, we construct a closed system of equations for the velocity $\boldsymbol{v}=\partial \boldsymbol{w}^{f} / \partial t$ in the liquid component and for the pressures $p$ and $q$, consisting of the modifications of Darcy's law (5.37) -(5.39) and boundary condition (5.40), in which we have $\boldsymbol{u}(\boldsymbol{x}, t)=0$, and of the equations

$$
p+\nu_{0} p_{*}^{-1} \frac{\partial p}{\partial t}=q, \quad \frac{1}{p_{*}} \frac{\partial p}{\partial t}+\operatorname{div}_{x} \boldsymbol{v}=0, \quad \boldsymbol{x} \in \Omega, \quad t>0 .
$$

We entitle the above described systems as Problem $F_{1}, F_{2}$, or $F_{3}$ depending on the forms of the matrices $B_{1}, B_{2}$, or $B_{3}$, having places in Darcy's laws.

II. Let $\mu_{1}<\infty$ and condition (2.5) holds true. We observe that the limiting displacements in the rigid skeleton are equal to zero. In order to find a more accurate asymptotic of the solution of the original model, we use again the re-normalization. Namely, let

$$
\boldsymbol{w}^{\varepsilon} \rightarrow \alpha_{\lambda} \boldsymbol{w}^{\varepsilon}
$$

Then new displacements satisfy the same problem as displacements before re-normalization, but with new parameters

$$
\alpha_{\eta} \rightarrow \alpha_{\eta} \alpha_{\lambda}^{-1}, \quad \alpha_{\lambda} \rightarrow 1, \quad \alpha_{\tau} \rightarrow \alpha_{\tau} \alpha_{\lambda}^{-1}
$$

Thus we arrive at the assumptions of Theorem 2.2. Namely, the limiting functions $\boldsymbol{u}(\boldsymbol{x}, t), \pi(\boldsymbol{x}, t), \Pi(\boldsymbol{x}, t, \boldsymbol{y})$, and $\boldsymbol{U}(\boldsymbol{x}, t, \boldsymbol{y})$ satisfy the system of micro- and macroscopic equations (5.5), (5.16), (5.10) and (5.17), in which the pressure $q$ is given by virtue of one of Problems $F_{1}-F_{3}$. The only difference from already considered case is in micro- and macroscopic continuity equations, because this equation depends on the value $\eta_{2}$. These micro- and macroscopic continuity equations coincide with Eq. (5.5) and Eq. (5.10) if we put there $\eta_{0}=\eta_{2}$.

Hence for $\boldsymbol{u}(\boldsymbol{x}, t)$ and $\pi(\boldsymbol{x}, t)$ there hold true the homogenized momentum equation in the form

$$
0=\operatorname{div}_{x}\left\{\mathbb{A}_{0}^{s}: \mathbb{D}(x, \boldsymbol{u})+B_{0}^{s} \operatorname{div}_{x} \boldsymbol{u}+B_{1}^{s} q-(q+\pi) \cdot \mathbb{I}\right\}+\hat{\rho} \boldsymbol{F}, \quad \boldsymbol{x} \in \Omega,
$$

continuity equation (5.23), in which we have $\eta_{0}=\eta_{2}$, and the boundary condition (2.10) .

The tensor $\mathbb{A}_{0}^{s}$, the matrices $C_{0}^{s}, B_{0}^{s}$ and $B_{1}^{s}$ and the constants $a_{0}^{s} \quad a_{1}^{s}$ are defined from Eqs. (5.30), (5.32) $-(5.33)$, in which we have $\eta_{0}=\eta_{2}$ and $\lambda_{0}=1$. 
III. If $\mu_{1}=\infty, p_{1}^{-1}, \eta_{1}^{-1}<\infty$ and $0<\lambda_{1}<\infty$ then re-normalizing by

$$
\boldsymbol{w}^{\varepsilon} \rightarrow \alpha_{\mu} \varepsilon^{-2} \boldsymbol{w}^{\varepsilon}
$$

we arrive at the assumptions of Theorem 2.2, when $\mu_{1}=1, \tau_{0}=0 \quad \lambda_{0}=\lambda_{1}$. Namely, functions $\boldsymbol{w}^{f}, p, \pi$ and $\boldsymbol{u}$ satisfy the following initial-boundary value problem in $\Omega_{T}$ :

$$
\left.\begin{array}{r}
\operatorname{div}_{x}\left\{\lambda_{1} \mathbb{A}_{0}^{s}: \mathbb{D}(x, \boldsymbol{u})+B_{0}^{s} \operatorname{div}_{x} \boldsymbol{u}+B_{1}^{s} p-(p+\pi) \cdot \mathbb{I}\right\}+\hat{\rho} \boldsymbol{F}=0, \\
\frac{\partial \boldsymbol{w}^{f}}{\partial t}=\frac{\partial \boldsymbol{u}}{\partial t}+B_{2}(1) \cdot\left(-\nabla p+\rho_{f} \boldsymbol{F}\right), \\
\frac{1}{p_{1}} p+\frac{1}{\eta_{1}} \pi+\operatorname{div}_{x} \boldsymbol{w}^{f}=(m-1) \operatorname{div}_{x} \boldsymbol{u}, \\
\frac{1}{\eta_{1}} \pi+C_{0}^{s}: \mathbb{D}(x, \boldsymbol{u})+a_{0}^{s} \operatorname{div}_{x} \boldsymbol{u}+a_{1}^{s} p=0 .
\end{array}\right\}
$$

As before, tensor $\mathbb{A}_{0}^{s}$, matrices $C_{0}^{s}, B_{0}^{s}$ and $B_{1}^{s}$ and constants $a_{0}^{s} \quad a_{1}^{s}$ are defined by formulas (5.30), (5.32) - (5.34), in which we have $\eta_{0}=\eta_{1}$ and $\lambda_{0}=\lambda_{1}$.

Note, that here $\nu_{0}=0$. Therefore the state equation $p+\nu_{0} p_{*}^{-1} \partial p / \partial t=q$ becomes $p=q$.

The problem is endowed by the corresponding homogeneous initial and boundary conditions.

\section{$\S 7$. Proof of Theorem 2.4}

7.1. Weak and two-scale limits of sequences of displacement and pressures.

On the strength of Theorem 2.1, the sequences $\left\{p^{\varepsilon}\right\},\left\{q^{\varepsilon}\right\},\left\{\pi^{\varepsilon}\right\}$ and $\left\{\boldsymbol{w}^{\varepsilon}\right\}$ are uniformly in $\varepsilon$ bounded in $L^{2}\left(\Omega_{T}\right)$. Then there exist a subsequence from $\{\varepsilon>0\}$ and functions $p, \pi, q$, and $\boldsymbol{w}$ such that as $\varepsilon \searrow 0$

$$
\boldsymbol{w}^{\varepsilon} \rightarrow \boldsymbol{w}, \quad p^{\varepsilon} \rightarrow p, \quad q^{\varepsilon} \rightarrow q, \quad \pi^{\varepsilon} \rightarrow \pi \quad L^{2}\left(\Omega_{T}\right)
$$

Moreover, since $\lambda_{0}, \mu_{0}>0$ then the bound (1.17) imply

$$
\nabla_{x} \boldsymbol{w}^{\varepsilon} \underset{\varepsilon \searrow 0}{\longrightarrow} \nabla_{x} \boldsymbol{w} \quad \text { weakly in } L^{2}\left(\Omega_{T}\right) .
$$

Due to limiting relations (7.1), (7.2) and Ngutseng's theorem, there exist one more subsequence from $\{\varepsilon>0\}$ and 1-periodic in $\boldsymbol{y}$ functions $P(\boldsymbol{x}, t, \boldsymbol{y})$, $\Pi(\boldsymbol{x}, t, \boldsymbol{y}), Q(\boldsymbol{x}, t, \boldsymbol{y})$, and $\boldsymbol{W}(\boldsymbol{x}, t, \boldsymbol{y})$ such that the sequences $\left\{p^{\varepsilon}\right\},\left\{\pi^{\varepsilon}\right\}$, $\left\{q^{\varepsilon}\right\}$ and $\left\{\nabla \boldsymbol{w}^{\varepsilon}\right\}$ two-scale converge as $\varepsilon \searrow 0$ respectively to $P, \Pi, Q$ and $\nabla_{x} \boldsymbol{w}+\nabla_{y} \boldsymbol{W}$. 


\subsection{Micro- and macroscopic equations.}

In the present section we do not consider functions of time $t$, which renormalize pressures. As we have shown before, we can ignore all functions of time.

Lemma 7.1. Two-scale limits of the sequences $\left\{p^{\varepsilon}\right\},\left\{\pi^{\varepsilon}\right\},\left\{q^{\varepsilon}\right\}$ and $\left\{\nabla \boldsymbol{w}^{\varepsilon}\right\}$ satisfy in $Y_{T}=Y \times(0, T)$ the following relations

$$
\begin{gathered}
\frac{1}{\eta_{0}} \Pi+(1-\chi)\left(d i v_{x} \boldsymbol{w}+d i v_{y} \boldsymbol{W}\right)=0 ; \\
\frac{1}{p_{*}} P+\chi\left(\operatorname{div}_{x} \boldsymbol{w}+\operatorname{div}_{y} \boldsymbol{W}\right)=0, \quad Q=P+\frac{\nu_{0}}{p_{*}} \frac{\partial P}{\partial t} ; \\
d i v_{y}\left(\chi \mu_{0}\left(\mathbb{D}\left(x, \frac{\partial \boldsymbol{w}}{\partial t}\right)+\mathbb{D}\left(y, \frac{\partial \boldsymbol{W}}{\partial t}\right)\right)+(1-\chi) \lambda_{0}(\mathbb{D}(x, \boldsymbol{w})+\mathbb{D}(y, \boldsymbol{W}))\right) \\
-\nabla_{y}(Q+\Pi)=0 .
\end{gathered}
$$

Lemma 7.2. The weak limits $p, \pi, q \boldsymbol{w}$ satisfy in $\Omega_{T}$ the following system of macroscopic equations:

$$
\begin{gathered}
\frac{1}{\eta_{0}} \pi+(1-m) d i v_{x} \boldsymbol{w}+\left\langle d i v_{y} \boldsymbol{W}\right\rangle_{Y_{s}}=0 ; \\
\frac{1}{p_{*}} p+m \operatorname{div}_{x} \boldsymbol{w}+\left\langle\operatorname{div}_{y} \boldsymbol{W}\right\rangle_{Y_{f}}=0, \quad q=p+\frac{\nu_{0}}{p_{*}} \frac{\partial p}{\partial t} ; \\
\tau_{0} \hat{\rho} \frac{\partial^{2} \boldsymbol{w}}{\partial t^{2}}=\operatorname{div}_{x}\left(\mu_{0}\left(m \mathbb{D}\left(x, \frac{\partial \boldsymbol{w}}{\partial t}\right)+\left\langle\mathbb{D}\left(y, \frac{\partial \boldsymbol{W}}{\partial t}\right)\right\rangle_{Y_{f}}\right)+\right. \\
\left.\lambda_{0}\left((1-m) \mathbb{D}(x, \boldsymbol{w})+\langle\mathbb{D}(y, \boldsymbol{W})\rangle_{Y_{s}}\right)-(q+\pi) \mathbb{I}\right)+\hat{\rho} \boldsymbol{F} .
\end{gathered}
$$

Proofs of these statements are the same as in lemmas 5.1-5.3.

\subsection{Homogenized equations.}

Lemma 7.3. Weak limits $p, \pi, q$ and $\boldsymbol{w}$ satisfy in $\Omega_{T}$ the following system of homogenized equations:

$$
\begin{aligned}
& \tau_{0} \hat{\rho} \frac{\partial^{2} \boldsymbol{w}}{\partial t^{2}}+\nabla(q+\pi)-\hat{\rho} \boldsymbol{F}= \\
& \operatorname{div}_{x}\left(\mathbb{A}_{2}: \mathbb{D}\left(x, \frac{\partial \boldsymbol{w}}{\partial t}\right)+\mathbb{A}_{3}: \mathbb{D}(x, \boldsymbol{w})+B_{4} d i v_{x} \boldsymbol{w}+\right. \\
& \left.\int_{0}^{t}\left(\mathbb{A}_{4}(t-\tau): \mathbb{D}(x, \boldsymbol{w}(\boldsymbol{x}, \tau))+B_{5}(t-\tau) \operatorname{div} v_{x} \boldsymbol{w}(\boldsymbol{x}, \tau)\right) d \tau\right), \\
& \frac{1}{p_{*}} p+m \operatorname{div}_{x} \boldsymbol{w}= \\
& -\int_{0}^{t}\left(C_{2}(t-\tau): \mathbb{D}(x, \boldsymbol{w}(\boldsymbol{x}, \tau))+a_{2}(t-\tau) \operatorname{div}_{x} \boldsymbol{w}(\boldsymbol{x}, \tau)\right) d \tau,
\end{aligned}
$$




$$
\begin{aligned}
& \frac{1}{\eta_{0}} \pi+(1-m) d i v_{x} \boldsymbol{w}= \\
&-\int_{0}^{t}\left(C_{3}(t-\tau): \mathbb{D}(x, \boldsymbol{w}(\boldsymbol{x}, \tau))+a_{3}(t-\tau) d i v_{x} \boldsymbol{w}(\boldsymbol{x}, \tau)\right) d \tau \\
& q=p+\frac{\nu_{0}}{p_{*}} \frac{\partial p}{\partial t} .
\end{aligned}
$$

Here $\mathbb{A}_{2}, \mathbb{A}_{3}$ and $\mathbb{A}_{4}$ - fourth-rank tensors, $B_{4}, B_{5}, C_{2}$ and $C_{3}$ - matrices and $a_{2}$ and $a_{3}$-scalars. The exact expressions for these objects are given below by formulas (7.26)-(7.31).

Proof. Let

$$
Z(\boldsymbol{x}, t)=\mu_{0} \mathbb{D}\left(x, \frac{\partial \boldsymbol{w}}{\partial t}\right)-\lambda_{0} \mathbb{D}(x, \boldsymbol{w}), \quad Z_{i j}=\mathbf{e}_{i} \cdot\left(Z \cdot \mathbf{e}_{j}\right), \quad z(\boldsymbol{x}, t)=\operatorname{div}_{x} \boldsymbol{w}
$$

As usual we look for the solution of the system of microscopic equations (7.3) - (7.5) in the form

$$
\begin{gathered}
\boldsymbol{W}=\int_{0}^{t}\left[\boldsymbol{W}^{0}(y, t-\tau) z(\boldsymbol{x}, \tau)+\sum_{i, j=1}^{3} \boldsymbol{W}^{i j}(\boldsymbol{y}, t-\tau) Z_{i j}(\boldsymbol{x}, \tau)\right] d \tau \\
P=\chi \int_{0}^{t}\left[P^{0}(\boldsymbol{y}, t-\tau) z(\boldsymbol{x}, \tau)+\sum_{i, j=1}^{3} P^{i j}(\boldsymbol{y}, t-\tau) Z_{i j}(\boldsymbol{x}, \tau)\right] d \tau \\
Q=\chi\left(Q_{0}(y) \cdot z(\boldsymbol{x}, t)+\sum_{i, j=1}^{3} Q_{0}^{i j}(\boldsymbol{y}) \cdot Z_{i j}(\boldsymbol{x}, t)+\right. \\
\left.\int_{0}^{t}\left[Q^{0}(\boldsymbol{y}, t-\tau) z(\boldsymbol{x}, \tau)+\sum_{i, j=1}^{3} Q^{i j}(\boldsymbol{y}, t-\tau) Z_{i j}(\boldsymbol{x}, \tau)\right] d \tau\right) \\
\Pi=(1-\chi) \int_{0}^{t}\left[\Pi^{0}(\boldsymbol{y}, t-\tau) z(\boldsymbol{x}, \tau)+\sum_{i, j=1}^{3} \Pi^{i j}(\boldsymbol{y}, t-\tau) Z_{i j}(\boldsymbol{x}, \tau)\right] d \tau
\end{gathered}
$$

where 1-periodic in $\boldsymbol{y}$ functions $\boldsymbol{W}^{0}, \boldsymbol{W}^{i j}, P^{0}, P^{i j}, Q_{0}, Q^{0}, Q^{i j}, Q_{0}^{i j}$, $\Pi^{0}, \Pi^{i j}$ satisfy the following periodic initial-boundary value problems in the elementary cell $Y$ : 


\section{Problem (I)}

$$
\begin{aligned}
& \operatorname{div}_{y}\left(\chi \left(\mu_{0} \mathbb{D}\left(y, \frac{\partial \boldsymbol{W}^{i j}}{\partial t}\right)+\right.\right. \\
& \left.(1-\chi)\left(\lambda_{0} \mathbb{D}\left(y, \boldsymbol{W}^{i j}\right)-\left((1-\chi) \Pi^{i j}+\chi Q^{i j}\right) \mathbb{I}\right)\right)=0 ; \\
& \frac{1}{p_{*}} P^{i j}+\chi \operatorname{div}_{y} \boldsymbol{W}^{i j}=0, \quad Q^{i j}=P^{i j}+\frac{\nu_{0}}{p_{*}} \frac{\partial P^{i j}}{\partial t}, \\
& \frac{1}{\eta_{0}} \Pi^{i j}+(1-\chi) \operatorname{div}_{y} \boldsymbol{W}^{i j}=0, \quad \boldsymbol{W}^{i j}(\boldsymbol{y}, 0)=\boldsymbol{W}_{0}^{i j}(\boldsymbol{y}) ; \\
& \operatorname{div}_{y}\left(\chi\left(\mu_{0} \mathbb{D}\left(y, \boldsymbol{W}_{0}^{i j}\right)+J^{i j}-Q_{0}^{i j} \mathbb{I}\right)\right)=0, \\
& \chi\left(Q_{0}^{i j}+\nu_{0} \operatorname{div}_{y} \boldsymbol{W}_{0}^{i j}\right)=0 .
\end{aligned}
$$

\section{Problem (II)}

$$
\begin{aligned}
& \operatorname{div}_{y}\left(\chi \left(\mu_{0} \mathbb{D}\left(y, \frac{\partial \boldsymbol{W}^{0}}{\partial t}\right)+\right.\right. \\
& \left.(1-\chi)\left(\lambda_{0} \mathbb{D}\left(y, \boldsymbol{W}^{0}\right)-\left((1-\chi) \Pi^{0}+\chi Q^{0}\right) \mathbb{I}\right)\right)=0 \\
& \chi\left(\frac{1}{p_{*}} P^{0}+\operatorname{div}_{y} \boldsymbol{W}^{0}+1\right)=0, \quad Q^{0}=P^{0}+\frac{\nu_{0}}{p_{*}} \frac{\partial P^{0}}{\partial t} \\
& (1-\chi)\left(\frac{1}{\eta_{0}} \Pi^{0}+\operatorname{div}_{y} \boldsymbol{W}^{0}+1\right)=0 \\
& \boldsymbol{W}^{0}(\boldsymbol{y}, 0)=\boldsymbol{W}_{0}^{0}(\boldsymbol{y}), \quad \operatorname{div}_{y}\left(\chi\left(\mu_{0} \mathbb{D}\left(y, \boldsymbol{W}_{0}^{0}\right)-Q_{0} \mathbb{I}\right)\right)=0 \\
& \chi\left(Q_{0}+\nu_{0}\left(\operatorname{div}_{y} \boldsymbol{W}_{0}^{0}+1\right)\right)=0 .
\end{aligned}
$$

Then

$$
\begin{gathered}
\mathbb{A}_{2}=\mu_{0} m \sum_{i, j=1}^{3} J^{i j} \otimes J^{i j}+\mu_{0} \mathbb{A}_{0}^{f} \\
\mathbb{A}_{0}^{f}=\sum_{i, j=1}^{3}\left\langle\left(\mu_{0} \mathbb{D}\left(y, \boldsymbol{W}_{0}^{i j}\right)\right\rangle_{Y_{f}} \otimes J^{i j}\right. \\
\mathbb{A}_{3}=\lambda_{0}(1-m) \sum_{i, j=1}^{3} J^{i j} \otimes J^{i j}-\lambda_{0} \mathbb{A}_{0}^{f}+\mu_{0} \mathbb{A}_{1}^{f}(0) \\
\mathbb{A}_{4}(t)=\mu_{0} \frac{d}{d t} \mathbb{A}_{1}^{f}(t)-\lambda_{0} \mathbb{A}_{1}^{f}(t) ; \\
\mathbb{A}_{1}^{f}(t)=\sum_{i, j=1}^{3}\left(\left\langle\mu_{0} \mathbb{D}\left(y, \frac{\partial \boldsymbol{W}^{i j}}{\partial t}(\boldsymbol{y}, t)\right)\right\rangle_{Y_{f}}+\left\langle\lambda_{0} \mathbb{D}\left(y, \boldsymbol{W}^{i j}(\boldsymbol{y}, t)\right)\right\rangle_{Y_{s}}\right) \otimes J^{i j}
\end{gathered}
$$




$$
\begin{gathered}
B_{5}(t)=\mu_{0}\left\langle\mathbb{D}\left(y, \frac{\partial \boldsymbol{W}^{0}}{\partial t}\right\rangle_{Y_{f}}+\lambda_{0}\left\langle\mathbb{D}\left(y, \boldsymbol{W}^{0}(\boldsymbol{y}, t)\right)\right\rangle_{Y_{s}} ;\right. \\
C_{2}(t)=-C_{3}(t)=\sum_{i, j=1}^{3}\left\langle\operatorname{div}_{y} \boldsymbol{W}^{i j}(\boldsymbol{y}, t)\right\rangle_{Y_{f}} J^{i j} \\
a_{2}(t)=-a_{3}(t)=\left\langle\operatorname{div}_{y} \boldsymbol{W}^{0}(\boldsymbol{y}, t)\right\rangle_{Y_{f}}, \quad B_{4}=\mu_{0}\left\langle\mathbb{D}\left(y, \boldsymbol{W}_{0}^{0}(\boldsymbol{y})\right)\right\rangle_{Y_{f}} .
\end{gathered}
$$

Lemma 7.4. Tensors $\mathbb{A}_{2}-\mathbb{A}_{4}$, matrices $B_{4}, B_{5}, C_{2}$ and $C_{3}$ and scalars $a_{2}$ and $a_{3}$ are well-defined and infinitely smooth in time.

If a porous space is connected, then the symmetric tensor $\mathbb{A}_{2}$ is strictly positively defined. For the case of disconnected porous space (isolated pores) $\mathbb{A}_{2}=0$ and the tensor $\mathbb{A}_{2}$ becomes strictly positively defined.

All these objects are well-defined if Problem $(I)$ and Problem(II) are well-posed. The solvability of above mentioned problems and smoothness with respect to time follow, due to linearity, from the standard a'priory estimates.

The symmetry of $\mathbb{A}_{2}$ proves in the same way as the symmetry of $\mathbb{A}_{0}$. If the porous space is disconnected, then the problem (7.19) has a unique solution linear in $\boldsymbol{y}$, such that

$$
\chi\left(\mathbb{D}\left(y, \boldsymbol{W}_{0}^{i j}\right)+J^{i j}\right)=0 .
$$

The last equality implies $\mathbb{A}_{2}=0$.

In this case the tensor $\mathbb{A}_{3}$ becomes strictly positively defined. Indeed

$$
\begin{gathered}
\mathbb{A}_{3}=\lambda_{0} \sum_{i, j=1}^{3} J^{i j} \otimes J^{i j}+\mu_{0} \mathbb{A}_{1}^{f}(0)= \\
\lambda_{0} \sum_{i, j=1}^{3} J^{i j} \otimes J^{i j}+\sum_{i, j=1}^{3}\left\langle\chi \mu_{0} \mathbb{D}\left(y, \frac{\partial \boldsymbol{W}^{i j}}{\partial t}(\boldsymbol{y}, 0)\right)+\frac{\lambda_{0}}{\mu_{0}} J^{i j}\right\rangle_{Y} \otimes J^{i j} .
\end{gathered}
$$

On the other hand, coming back to (7.17) at initial time moment we see that

$$
\begin{gathered}
\left\langle\chi \mu_{0} \mathbb{D}\left(y, \frac{\partial \boldsymbol{W}^{i j}}{\partial t}(\boldsymbol{y}, 0)\right): \mathbb{D}\left(y, \boldsymbol{W}_{0}^{k l}\right)\right\rangle_{Y}= \\
-\lambda_{0}\left\langle\chi \mathbb{D}\left(y, \boldsymbol{W}_{0}^{i j}\right): \mathbb{D}\left(y, \boldsymbol{W}_{0}^{k l}\right)\right\rangle_{Y}-\left.\left\langle\frac{1}{\eta_{0}} \Pi^{i j} \cdot \Pi^{k l}\right\rangle_{Y}\right|_{t=0} .
\end{gathered}
$$

Moreover, due to (7.32)

$$
\left\langle\chi \mu_{0} \mathbb{D}\left(y, \frac{\partial \boldsymbol{W}^{i j}}{\partial t}(\boldsymbol{y}, 0)\right): \mathbb{D}\left(y, \boldsymbol{W}_{0}^{k l}\right)\right\rangle=-\left\langle\chi \mathbb{D}\left(y, \frac{\partial \boldsymbol{W}^{i j}}{\partial t}(\boldsymbol{y}, 0)\right): J^{k l}\right\rangle_{Y}
$$

which proves our statement. 


\section{References}

[1] Coussy O. Poromechanics. John Wiley and Sons, Chichester, 2004.

[2] Burridge R., Keller J. B. Poroelasticity equations derived from microstructure // J. Acoust. Soc. Am. 1981. V. 70. N4. P. 1140-1146.

[3] Sanchez-Palencia E., Non-Homogeneous Media and Vibration Theory, Lecture Notes in Physics, Vol.129, (1980), Springer, Berlin.

[4] Gilbert R. P., Mikelić A. Homogenizing the acoustic properties of the seabed: Part I // Nonlinear Analysis. 2000. V. 40. P. 185-212.

[5] Biot $M$. Generalized theory of acoustic propagation in porous dissipative media // J. Acoust. Soc. Am. 1962. V. 34. P. 1256-1264.

[6] Clopeau Th., Ferrin J. L., Gilbert R. P., Mikelić A. Homogenizing the acoustic properties of the seabed: Part II // Mathematical and Computer Modelling. 2001. V. 33. P. 821-841.

[7] Ferrin J. L., Mikelić A. Homogenizing the acoustic properties of a porous matrix containing an incompressible inviscid fluids // Math. Meth. Appl. Sci. 2003. V. 26. P. 831-859.

[8] Nguetseng G. A general convergence result for a functional related to the theory of homogenization // SIAM J. Math. Anal. 1989. V. 20. P. $608-623$.

[9] Nguetseng G. Asymptotic analysis for a stiff variational problem arising in mechanics // SIAM J. Math. Anal. 1990. V. 21. P. 1394-1414.

[10] Lukkassen, D., Nguetseng, G., Wall P. Two-scale convergence // Int. J. Pure and Appl. Math. 2002. V. 2. N1. P. 35-86.

[11] Bakhvalov N. S. and Panasenko G. P. Averaging Processes in Periodic Media [in Russian], Nauka, Moscow (1984).

[12] Acerbi E., Chiado Piat V., Dal Maso G., Percivale D. An extension theorem from connected sets and homogenization in general periodic domains // Nonlinear Anal. 1992. V. 18. P. 481-496.

[13] Jikov V. V., Kozlov S. M., and Oleinik O. A. Homogenization of Differential Operators and Integral Functionals, Springer-Verlag, New York, 1994.

[14] Ladyzhenskaya O.A. The mathematical Theory of Viscous Incompressible Flow, Gordon and Breach, New York, 1969. 
Anvarbek Meirmanov

Ugra State University, Khanti-Mansiisk, Russia;

Center for Advanced Mathematics and Physics, Electrical and Mechanical Engineering College, National University of Science and Technology, Peshawar Road, Rawalpindi, Pakistan;

email: anvarbek@list.ru 